TI 2016-069/IV

Tinbergen Institute Discussion Paper
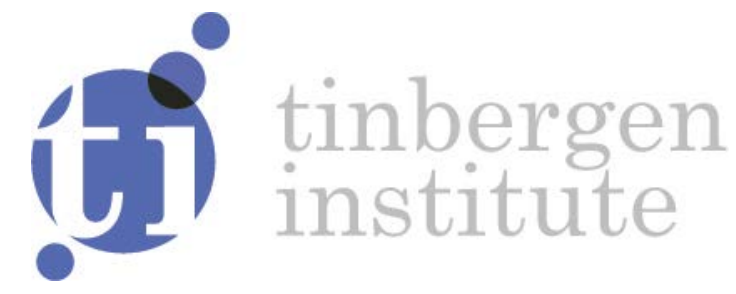

Fractional Integration and Fat Tails for Realized Covariance Kernels and Returns

REVISION: JUNE 2017

André Lucas ${ }^{1}$

Anne Opschoor ${ }^{1}$

Vrije Universiteit Amsterdam and Tinbergen Institute, The Netherlands 
Tinbergen Institute is the graduate school and research institute in economics of Erasmus University Rotterdam, the University of Amsterdam and VU University Amsterdam.

Contact: discussionpapers@tinbergen.nl

More TI discussion papers can be downloaded at http://www.tinbergen.nl

Tinbergen Institute has two locations:

Tinbergen Institute Amsterdam

Gustav Mahlerplein 117

1082 MS Amsterdam

The Netherlands

Tel.: +31(0)205984580

Tinbergen Institute Rotterdam

Burg. Oudlaan 50

3062 PA Rotterdam

The Netherlands

Tel.: +31(0)10408 8900 


\title{
Fractional Integration and Fat Tails for Realized Covariance Kernels and Returns
}

\author{
Anne Opschoor ${ }^{a *}$ and André Lucas ${ }^{a}$ \\ ${ }^{a}$ Vrije Universiteit Amsterdam and Tinbergen Institute
}

This version: June 29, 2017

\begin{abstract}
We introduce a new fractionally integrated model for covariance matrix dynamics based on the long-memory behavior of daily realized covariance matrix kernels and daily return observations. We account for fat tails in both types of data by appropriate distributional assumptions. The covariance matrix dynamics are formulated as a numerically efficient matrix recursion that ensures positive definiteness under simple parameter constraints. Using intraday stock data over the period 2001-2012, we construct realized covariance kernels and show that the new fractionally integrated model statistically and economically outperforms recent alternatives such as the Multivariate HEAVY model and the multivariate HAR model. In addition, the long-memory behavior is more important during non-crisis periods.
\end{abstract}

Keywords: multivariate volatility; fractional integration; realized covariance matrices; heavy tails; matrix- $F$ distribution; score dynamics.

${ }^{*}$ Corresponding author, e-mail address: a.opschoor@vu.nl. Postal address: Vrije Universiteit Amsterdam, De Boelelaan 1105, 1081 HV, Amsterdam, The Netherlands. Phone number: +31(0)20-5982663. 


\section{Introduction}

Various fields in financial econometrics, such as risk- and portfolio management, require the use of an adequate multivariate volatility model to estimate or forecast the covariance matrix of financial asset returns. We can distinguish two main lines of literature dealing with these models, namely multivariate generalized autoregressive conditional heteroskedasticity (GARCH) type models (for an overview, see Silvennoien and Teräsvirta, 2009) and stochastic volatility (SV) type models (for an overview, see Asai et al., 2006). More recently, the availability of intraday high-frequency data has led to a new class of volatility models including realized (co)variance measures. Such realized measures help to describe and forecast volatility more precisely than traditional measures such as squares and crossproducts of daily returns; see for instance Andersen et al. (2001). Typically, either either realized variance measures (Barndorff-Nielsen and Shephard, 2002) or realized kernel measures (Barndorff-Nielsen et al., 2008) are included. Examples of the former include the Wishart autoregressive (WAR) model of Gourieroux et al. (2009) and the Conditional Autoregressive Wishart (CAW) model of Golosnoy et al. (2012), while examples of the latter include the High-frEquency-bAsed VolatilitY (HEAVY) model of Shephard and Sheppard (2010) and its multivariate extension by Noureldin et al. (2012), and the multiplicative error model (MEM) model of Englo and Gallo (2006).

Volatilities are typically strongly persistent, which has led to the introduction of volatility models with long-memory features. A seminal reference is the fractionally integrated GARCH (FI-GARCH) model of Baillie et al. (1996), which is based on squared daily returns. Realized variance measures exhibit even stronger long memory features than squared daily returns. Andersen et al. (2001) for instance find that realized measures are highly persistent and behave as fractionally integrated processes that can be modeled by autoregressive fractionally integrated moving average (ARFIMA) models; see also Koopman et al. (2005). Corsi (2009) also caputures long-memory volatility dynamics, but does so using a heterogeneous autoregressive (HAR) model, which relates realized volatility to a linear combination of lagged daily, weekly and monthly realized volatilities. Proietti (2016) introduces an alternative integrated moving average model for realized variances and assesses its predictive power against other univariate models. 
The univariate volatility models with long-memory features available in the literature have two main drawbacks complicating their application in a multivariate context. First, these models do not account for fat-tailed returns and outliers in either the realized measures, the returns, or both. Though fat-tailed distributions are often used to describe returns, thintailed distributions such as the Wishart are typically used for the realized measures despite the fact that also data for the realized measures can be subject to outliers and influential observations. For example, the Flash Crash in 2010 led to a spike in the realized (co)variance of a large number of assets. Ignoring the possible occurrence of such influential events in the specification of the volatility propagation mechanism and the likelihood function can have a huge impact on the estimated volatility dynamics for each of the volatility models discussed above. Second, multivariate models that incorporate the long-memory features of (realized) (co)variances face the challenge to simultaneously avoid the curse of dimensionality and solve the requirement of ensuring positive definite covariance matrices. Chiriac and Voev (2011) deal with these issues by proposing vector ARFIMA (VARFIMA) models for the cholesky decomposition of the realized covariance matrix. The same study also extend the HAR model of Corsi (2009) to the multivariate setting. Bauer and Vorkink (2011) solve the issue differently by modeling the matrix-logarithm of the realized covariance matrix. Both studies, however, model the vectorized (vech) matrix of interest. This may become computationally intensive in higher dimensions. Moreover, neither model accounts for the possible fat-tailedness of realized measures and its impact on the volatility dynamics as discussed earlier.

In this paper, we solve both of the above issues by introducing a new multivariate volatility model for realized (kernel) covariance matrices. The model can also be used to simultaneous model realized covariance matrices and daily returns. Our model allows for both the long-memory behavior and the fat-tailedness of (realized) covariances and returns by combining fractionally integrated dynamics with the generalized autoregressive score (GAS) dynamics of Creal et al. (2011, 2013). The only paper to our knowledge that combines long-memory and GAS is Janus et al. (2014), but this paper is set entirely in a univariate context and does not incorporate realized measures. Moreover, Janus et al. fails to provide parameter constraints to ensure positive definiteness of the covariance matrix. The model set-up developed in the remainder of this paper, by contrast, has easy parameter 
restrictions that render the covariance matrix positive definite at all times.

To account for fat tails, we assume a matrix- $F$ distribution for the realized covariance matrix and a Student's $t$ distribution for the daily returns. The use of the matrix- $F$ distribution for realized volatility models was first propagated in Opschoor et al. (2017). The combination of the matrix- $F$ and vector-valued Student's $t$ distribution allows for a tractable analytic expression for the score of the log predictive density with respect to the unknown, dynamic covariance matrix. The score expressions can be used in the generalized autoregressive score-driven (GAS) framework of Creal et al. (2013) to provide the covariance matrix dynamics. The score-driven framework has been applied successfully in a number of other settings, including volatility and location modeling (Harvey, 2013; Harvey and Luati, 2014), credit risk modeling (Creal et al., 2014), and systemic risk modeling (Oh and Patton, 2016; Lucas et al., 2014). The availability of a closed-form expression for the likelihood function and the optimality of score-driven steps (see Blasques et al., 2015) make the GAS framework a good starting point for combining long-memory, fat tails, robust time-varying parameter dynamics, and ease of estimation. As we show later, the score expressions automatically account for a reduced impact of outlying realized covariance matrices and/or return vectors in an intuitive way. The convenient advantage of the score-driven approach is also that we can directly apply the theoretical results of Conrad and Haag (2006) to obtain simple parameter restrictions to establish positive definiteness of the estimated covariance matrices over the entire sample period. In addition, due to the matrix formulation of our volatility dynamics, the introduction of the long-memory feature can be done in a parsimonious yet flexible way that allows for generalizations of the model in many directions of empirical interest. The parsimony of the approach is a major asset in the multivariate context, where the curse of dimensionality looms large.

We provide an empirical application of our multivariate Fractionally Integrated model based on the matrix- $F$ and Student's $t$ distribution (FIGAS tF model from now on) on daily realized kernels and daily returns for 15 equities from the S\&P 500 index. Our sample spans the period January 2001 to December 2012. Using a forecasting horizon of 1, 5, 10, and 22 days ahead, we compare both statistically and economically the performance of our new dynamic covariance matrix model to several strong benchmarks, such as the HEAVY model (Noureldin et al., 2012), the GAS tF model (Opschoor et al., 2017) and 
the multivariate extension of the HAR model of Corsi (2009). Using a quasi-likelihood loss function, the FIGAS model outperforms the competing HEAVY and HAR models, both inside and outside crisis periods. Interestingly, we find that the fractionally integrated part of our new model only outperforms the short-memory GAS model during non-crisis periods. Hence the long-memory property seems particularly relevant during calm periods. We assess the economic significance of our results by considering mean-variance efficient portfolios based on the forecasts. Again we find that the FIGAS tF model outperforms its competitors by producing statistically significantly lower ex-post conditional portfolio standard deviations.

The rest of this paper is set up as follows. In Section 2, we introduce the new FIGAS $\mathrm{tF}$ model for realized covariance matrices and return vectors under fat-tails. In Section 3 we provide a simulation experiment to show the performance of the model and estimation procedure. In Section 4 we apply the model to a panel of daily realized kernels and equity returns. We conclude in Section 5.

\section{Modeling Framework}

\subsection{The fractionally integrated GARCH model}

Before we introduce our new score-driven fractionally integrated volatility model, we first briefly recapitulate the main steps in the development of the univariate fractionally integrated $\operatorname{GARCH}(1,1)$ or FIGARCH$(1, d, 1)$ model of Baillie et al. (1996). This paves the way to the new fractional integrated dynamics in the score-driven framework. The FIGARCH $(1, d, 1)$ model is obtained by rewriting the standard $\operatorname{GARCH}(1,1)$ model of Bollerslev (1986) as

$$
\sigma_{t+1}^{2}=\omega+\alpha \epsilon_{t}^{2}+\beta \sigma_{t}^{2} \quad \Leftrightarrow \quad(1-\alpha L-\beta L) \epsilon_{t+1}^{2}=\omega+(1-\beta L) v_{t+1}
$$

with $L$ the lag operator $L \sigma_{t+1}^{2}=\sigma_{t}^{2}, \sigma_{t}^{2}$ the conditional variance of $\epsilon_{t}$, and $v_{t}=\epsilon_{t}^{2}-\sigma_{t}^{2}$ a martingale difference. Baillie et al. (1996) introduce the $\operatorname{FIGARCH}(1, d, 1)$ model by replacing the left-hand side lag polynomial $(1-\alpha L-\beta L)$ by $(1-L)^{d}(1-\phi L)$, with $|\phi|<1$ 
and $(1-L)^{d}$ the fractional difference operator defined by the binomial expansion

$$
(1-L)^{d}=1-d L+\frac{d(d-1)}{2 !} L^{2}-\frac{d(d-1)(d-2)}{3 !} L^{3}+\ldots
$$

for any real order of fractional integration $d>-1$. Using $v_{t}=\epsilon_{t}^{2}-\sigma_{t}^{2}$, the $\operatorname{FIGARCH}(1, d, 1)$ model can also be rewritten in its $\operatorname{ARCH}(\infty)$ representation

$$
(1-L)^{d}(1-\phi L) \epsilon_{t+1}^{2}=\omega+(1-\beta L) v_{t+1} \quad \Leftrightarrow \quad \sigma_{t+1}^{2}=\tilde{\omega}+\Psi(L) \epsilon_{t+1}^{2}
$$

with $\tilde{\omega}=1 /(1-\beta)$ and

$$
\Psi(L)=1-\frac{(1-L)^{d}(1-\phi L)}{(1-\beta L)}=\sum_{i=1}^{\infty} \psi_{i} L^{i}
$$

Thus, the conditional variance $\sigma_{t+1}^{2}$ depends on lags of $\epsilon_{t+1}^{2}$, where the weight assigned to each lag declines hyperbolically according to $\Psi(L)$.

\subsection{Score-driven fractionally integrated volatility dynamics}

Analogously to the FIGARCH case, we can now introduce the fractionally integrated scoredriven multivariate volatility model. Consider a $(k \times 1)$ vector process $y_{t}$ and a $(k \times k)$ matrix process $R K_{t}, t=1, \ldots, T$, generated by

$$
\begin{aligned}
y_{t} & =\mu+V_{t}^{1 / 2} z_{t}, & z_{t} \mid \mathcal{F}_{t-1} & \sim D_{z}\left(0, \mathrm{I}_{k}\right), \\
R K_{t} & =V_{t}^{1 / 2} Z_{t}\left(V_{t}^{1 / 2}\right)^{\prime}, & Z_{t} \mid \mathcal{F}_{t-1} & \sim D_{Z}\left(\mathrm{I}_{k}\right),
\end{aligned}
$$

where $\mathcal{F}_{t-1}$ is the information set containing all information up to time $t-1, \mu$ denotes the conditional mean vector of the return vector $y_{t}, V_{t}$ denotes the conditional covariance matrix, $R K_{t}$ denotes the realized kernel covariance matrix measure, and $z_{t}$ and $Z_{t}$ denote a $(k \times 1)$ vector-valued and $(k \times k)$ matrix-valued innovation. The matrix root $V_{t}^{1 / 2}$ is defined such that $V_{t}^{1 / 2}\left(V_{t}^{1 / 2}\right)^{\prime}=V_{t}$. The realized kernel process $R K_{t}$ is a consistent and robust estimator of $V_{t}$ correcting for market-microstructure noise; for more details, see BarndorffNielsen et al. (2011). For simplicity and ease of notation, we set $\mu=0$. Note, however, 
that we can easily allow for time-varying conditional means $\mu_{t}$ that incorporate for example autoregressive or moving average dynamics into the specification of $y_{t}$.

We assume $V_{t}$ follows the score-driven dynamics as introduced by Creal et al. $(2011,2013)$ and Harvey (2013). Score dynamics adjust the time varying parameter $V_{t}$ in the direction of steepest ascent of the local log likelihood function. The approach is computationally easy to implement given its explicit form for the likelihood function. Score-driven dynamics also possess information theoretic optimality properties; see Blasques et al. (2015). Let $p\left(y_{t}, R K_{t} \mid V_{t}\right)$ denote the predictive conditional density for $\left(y_{t}, R K_{t}\right)$. Then the scoredriven dynamics for $V_{t}$ are driven by the scaled score

$$
s_{t}=S_{t} \cdot\left(\partial \log p\left(y_{t}, R K_{t} \mid V_{t}\right) / \partial V_{t}\right) \cdot S_{t}^{\prime}
$$

where $S_{t}$ is a scaling matrix to correct for the curvature of the log predictive density at time $t$. We come back to the precise form of the conditional observation density $p\left(y_{t}, R K_{t} \mid V_{t}\right)$ and the scaled score $s_{t}$ in Subsection 2.3. For now, it suffices to note that our distributional and scaling choices allow us to write $s_{t}$ as $s_{t}=s_{t}^{\star}-V_{t}$, where $s_{t}^{\star}$ is positive definite for all $t$.

To introduce fractionally integrated dynamics for the score-driven model, we first note that due to the standard properties of a predictive density score, $s_{t}$ is a martingale difference by construction. It thus automatically takes the role of the martingale difference $v_{t}$ in (1). Similarly, $s_{t}^{\star}$ is always positive definite and takes the role of $\epsilon_{t}^{2}$ in (1). In particular, if we assume $y_{t}$ to be conditionally normally distributed and we scale the score by the inverse Fisher information matrix, we obtain $s_{t}=y_{t} y_{t}^{\prime}-V_{t}$, such that $s_{t}^{\star}=y_{t} y_{t}^{\prime}$, which is the familiar expression for a multivariate GARCH model. Consider the standard GAS $(1,1)$ dynamics $V_{t+1}=\Omega+\alpha^{*} s_{t}+\beta^{*} V_{t}$ of Creal et al. (2013), where $\alpha^{*}$ and $\beta^{*}$ are scalar parameters. Using $V_{t}=s_{t}^{\star}-s_{t}$ and the GAS $(1,1)$ model, the fractionally integrated score-driven dynamics can now be derived analogously to the FIGARCH setting, namely

$$
\begin{aligned}
V_{t+1}=\Omega+\alpha^{*} s_{t}+\beta^{*} V_{t} & \Leftrightarrow\left(1-\beta^{*} L\right) s_{t+1}^{\star}=\Omega+\left(1+\alpha^{*} L-\beta^{*} L\right) s_{t+1} \\
& \Rightarrow(1-L)^{d}(1-\phi L) s_{t+1}^{\star}=\Omega+(1-\beta L) s_{t+1},
\end{aligned}
$$

where we again replaced the standard GAS polynomial $\left(1-\beta^{*} L\right)$ by the fractionally inte- 
grated polynomial $(1-L)^{d}(1-\phi L)$, and defined $\beta=\beta^{*}-\alpha^{*}$ to homogenize the notation with the FIGARCH case, where $\phi$ and $\beta$ are scalar parameters, and $\Omega$ is a fixed positive definite parameter matrix. We label the model the fractionally integrated generalized autoregressive score model of order $(1, d, 1)$, or in short FIGAS $(1, d, 1)$. Using the definition $s_{t}=s_{t}^{\star}-V_{t}$, we can rewrite (8) as

$$
V_{t+1}=\frac{\Omega}{1-\beta}+\left(1-\frac{(1-L)^{d}(1-\phi L)}{1-\beta L}\right) s_{t+1}^{\star}=\tilde{\Omega}+\Psi(L) s_{t+1}^{\star},
$$

with $\tilde{\Omega}=\Omega /(1-\beta)$, and $\Psi(L)$ as defined in (4). Thus, also for the FIGAS $(1, d, 1)$ model, the conditional covariance matrix $V_{t+1}$ is an infinite weighted sum of current and past $s_{t}^{\star}$, where the weight assigned to each lag declines hyperbolically according to $\Psi(L)$.

The fractional integrated score dynamics introduced in (8) are substantially different from those introduced in Janus et al. (2014). Whereas Janus et al. impose a fractional polynomial directly on the (in their case univariate) volatility parameter $V_{t}$, we follow the original approach of Baillie et al. (1996) much more closely and impose the fractional polynomial on $s_{t}^{\star}$. An important advantage of our current FIGAS specification compared to that of Janus et al. (2014) is not only that we allow for a multivariate setting, but also that we can immediately establish the positive definiteness of the sequence of covariance matrices $V_{t}$ for all times $t$ using simple parameter restrictions. This is stated in the following proposition for the FIGAS $(1, d, 0)$ model, which we use in the empirical application later on.

Proposition 1 Assume that $\Omega$ and $s_{t}^{\star}$ in (8) are positive definite for all $t$. Then the conditional covariance matrices $V_{t}$ from the FIGAS $(1, d, 0)$ model are positive definite if

Case 1: $\quad 0<\beta<1, \quad d-\beta \geq 0$;

Case 2: $\quad-1<\beta<0, \quad(d-\sqrt{2(2-d)}) / 2 \leq \beta$.

The proof follows directly from Corollary 3 of Conrad and Haag (2006). The proposition is stated for the FIGAS $(1, d, 0)$ model, which is the model we use in the empirical application later on. It is straightforward, however, to apply the results of Conrad and Haag (2006) also for more general forms of the FIGAS model, such as the FIGAS $(1, d, 1)$. This feature is quite convenient and follows from the way we have set up the fractionally integrated dynamics in contrast to earlier papers on fractionally integrated score-driven dynamics. 
The conditions of Proposition 1 are easily checked for our model. The assumption on $\Omega$ is easily enforced through the model's parameterization. We show in the next subsection that also the second assumption on the positive definiteness of $s_{t}^{\star}$ is automatically satisfied for the fat-tailed distributional choices made in this paper. The restrictions in case 1 or case 2 of the proposition can then again be easily imposed by the model's parameterization.

\subsection{Score for the Student's $t-$ matrix- $F$ distribution}

We now turn to our choice for the conditional observation densities $D_{z}(\cdot)$ and $D_{Z}(\cdot)$ in (5)-(6) to complete the FIGAS specification under fat tails. To account for the possible fattailedness of the returns, we assume that $y_{t}$ follows a (conditional) Student's $t$ distribution,

$$
p_{y}\left(y_{t} \mid V_{t}, \mathcal{F}_{t-1} ; \nu_{0}\right)=\frac{\Gamma\left(\left(\nu_{0}+k\right) / 2\right)}{\Gamma\left(\nu_{0} / 2\right)\left[\left(\nu_{0}-2\right) \pi\right]^{k / 2}\left|V_{t}\right|^{1 / 2}} \times\left(1+\frac{y_{t} V_{t}^{-1} y_{t}}{\nu_{0}-2}\right)^{-\left(\nu_{0}+k\right) / 2}
$$

with degrees of freedom parameter $\nu_{0}>2$ and $V_{t}$ a positive definite covariance matrix at time $t$. Similarly, to account for possible fat tails of the realized kernel distribution, we assume that $R K_{t}$ has a matrix- $F$ distribution. The use of a matrix- $F$ distribution for realized measures was first proposed in Opschoor et al. (2017). The matrix- $F$ distribution is given by

$$
p_{R K}\left(R K_{t} \mid V_{t}, \mathcal{F}_{t-1} ; \nu_{1}, \nu_{2}\right)=K\left(\nu_{1}, \nu_{2}\right) \times \frac{\left|\frac{\nu_{1}}{\nu_{2}-k-1} V_{t}^{-1}\right|^{\frac{\nu_{1}}{2}}\left|R K_{t}\right|^{\left(\nu_{1}-k-1\right) / 2}}{\left|\mathrm{I}_{k}+\frac{\nu_{1}}{\nu_{2}-k-1} V_{t}^{-1} R K_{t}\right|^{\left(\nu_{1}+\nu_{2}\right) / 2}},
$$

with positive definite expectation $\mathbb{E}_{t}\left[R K_{t} \mid \mathcal{F}_{t-1}\right]=V_{t}$, and degrees of freedom parameters $\nu_{1}, \nu_{2}>k+1$, where

$$
K\left(\nu_{1}, \nu_{2}\right)=\frac{\Gamma_{k}\left(\left(\nu_{1}+\nu_{2}\right) / 2\right)}{\Gamma_{k}\left(\nu_{1} / 2\right) \Gamma_{k}\left(\nu_{2} / 2\right)}
$$

and $\Gamma_{k}(x)$ is the multivariate Gamma function

$$
\Gamma_{k}(x)=\pi^{k(k-1) / 4} \cdot \prod_{i-1}^{k} \Gamma(x+(1-i) / 2)
$$


see for example Konno (1991). Both observation densities depend on the common time varying covariance matrix $V_{t}$. We assume that conditional on $V_{t}$ and $\mathcal{F}_{t-1}$, returns $y_{t}$ and realized covariances $R K_{t}$ are independent such that the joint conditional density $p\left(y_{t}, R K_{t} \mid V_{t}\right)$ is the product of the conditional marginal densities (10) and (11). Preliminary data analyses for bivariate snapshots of the data reveal that this assumption is empirically reasonable: conditional correlations between returns and realized kernels are typically small.

Given the two observation densities (10) and (11), we have the following result. The proof can be found in the appendix.

Proposition 2 Using the scaling matrix $S_{t}=\sqrt{2} V_{t}$, the scaled score for the Student's $t$ matrix-F distribution equals

$$
s_{t}=\frac{1}{\nu_{1}+1} \cdot w_{t} \cdot y_{t} y_{t}^{\prime}+\frac{\nu_{1}}{\nu_{1}+1} \cdot W_{t} \cdot R K_{t}-V_{t}
$$

with weight $w_{t}$ and weight matrix $W_{t}$ given by

$$
\begin{aligned}
w_{t} & =\left(\nu_{0}+k\right) \cdot\left(\nu_{0}-2+y_{t}^{\prime} V_{t}^{-1} y_{t}\right)^{-1} \\
W_{t} & =\frac{\nu_{1}+\nu_{2}}{\nu_{2}-k-1} \cdot\left(\mathrm{I}_{k}+\frac{\nu_{1}}{\nu_{2}-k-1} R K_{t} \cdot V_{t}^{-1}\right)^{-1} .
\end{aligned}
$$

Finally, given $\nu_{0}, \nu_{1}, \nu_{2}>0$ and $V_{t}$ and $R K_{t}$ positive definite, we have that $s_{t}^{\star}=s_{t}+V_{t}$ is positive semi-definite.

The score $s_{t}$ has a number of intuitive properties. It contains familiar terms of the form $w_{t} \cdot y_{t} y_{t}^{\prime}$ and $W_{t} \cdot R K_{t}$. For the normal distribution, $w_{t} \equiv 1$, such that $V_{t+1}$ directly reacts to the unweighted squared daily returns $y_{t} y_{t}^{\prime}$, similar as in a standard multivariate GARCH model. Analogously, for the Wishart distribution $W_{t}=\mathrm{I}_{k}$, such that $V_{t}$ reacts directly to the realized kernel value. For finite $\nu_{0}$ and $\nu_{2}$, the weights $w_{t}$ and $W_{t}$ automatically downplay the importance of outlying values of $y_{t}$ and $R K_{t}$, respectively. For example, if $y_{t} V_{t}^{-1} y_{t}$ grows large, the weight $w_{t}$ tends to zero. The same holds for $W_{t}$ if $R K_{t} V_{t}^{-1}$ grows large. The presence of both $w_{t}$ and $W_{t}$ thus gives the model a doubly robust feature for both types of measurements for $V_{t}$. The parameter $\nu_{1}$ in (14) determines the relative weights in the score attributed to $y_{t} y_{t}^{\prime}$ and $R K_{t}$. A larger value of $\nu_{1}$ decrease the variance of the matrix- $F$ 
distribution and indicates that $R K_{t}$ is a more reliable estimate of $V_{t}$. As a result, more weight is put on the score related to $R K_{t}$ than on the score related to $y_{t}$ if $\nu_{1}$ increases.

Important for the fractionally integresed dynamics of Subsection 2.2 is that $s_{t}^{\star}=s_{t}+V_{t}$ is positive semi-definite for all $t$. Looking at the expressions in Proposition 2 this requires the degrees of freedom parameters to be strictly positive and $V_{t}$ to be positive definite. Then the weight $w_{t}$ is clearly positive, and $w_{t} \cdot y_{t} y_{t}$ is positive semi-definite. We can easily show ${ }^{1}$ that also the term $W_{t} \cdot R K_{t}$ is positive semi-definite in that case. This allows us to conclude that the parameter restrictions formulated in Proposition 1 can be used to enforce positive definiteness of $V_{t}$ for all $t$ in the FIGAS model.

\subsection{Estimation}

We estimate the parameters of the FIGAS model by maximum likelihood. In order to circumvent the number of estimated parameters corresponding to $\tilde{\Omega}=\Omega /(1-\beta)$, we target $\tilde{\Omega}$ by $\tilde{\Omega}=\left(1-\sum_{i=1}^{\infty} \psi_{i}\right)(1 / T) \sum_{t=1}^{T} R K_{t}$ with $\psi_{i}$ defined in (4). This specification is related to the covariance targeting approach as $\Omega$ depends on the sample average of $R K_{t}$. We estimate the remaining static parameter vector $\theta=\left\{\phi^{*}, \beta, \nu_{0}, \nu_{1}, \nu_{2}, d\right\}$ of the FIGAS model by maximum likelihood. To do so, we maximize the log-likelihood $\mathcal{L}_{\mathrm{tF}}(\theta)=\sum_{t=1}^{T} \mathcal{L}_{t}$, where $\mathcal{L}_{t}$ is defined as the sum of the log likelihoods of the Student's $t$ and the matrix- $F$ distributions of equations (10) and (11). This standard prediction error decomposition of the likelihood function is made possible due to the observation-driven nature of the FIGAS model using the classification of Cox (1981). The starting value $V_{1}$ can be either estimated or set equal to $R K_{1}$.

The maximum likelihood estimation for the fractionally integrated model requires truncation of the infinite distributed lags in (2). We choose the maximum number of lags, which equals $T-1$. Finally we put the pre-sample innovations equal to zero, guided by the finding of Bollerslev and Mikkelsen (1996) that the effect of pre-sample values has a negligible effect on the parameter estimates, provided that the sample size is sufficiently large. Pre-sample values of $V_{t}$ are put to the sample analogue of the unconditional covariance matrix.

\footnotetext{
${ }^{1}$ Note that $W_{t} \cdot R K_{t}$ can be written as $\frac{\nu_{1}+\nu_{2}}{\nu_{2}-k-1}\left(R K_{t}^{-1}+\frac{\nu_{1}}{\nu_{2}-k-1} V_{t}^{-1}\right)^{-1}$, which is positive definite as $R K_{t}$ and $V_{t}$ (and their inverses) are both positive definite, $\nu_{1}, \nu_{2}>k+1$, and the inverse of the sum of two positive definite matrices is again positive definite.
} 
Table 1: Parameter estimations of FIGAS tF DGP

This table shows Monte Carlo averages and standard deviations (in parentheses) of parameter estimates from simulated FIGAS tF processes with $\tilde{\Omega}=c \mathrm{I}$. The table reports the mean and the standard deviation in parentheses based on 1000 replications.

\begin{tabular}{crrr}
\hline \multicolumn{1}{c}{ Coef. } & True & \multicolumn{1}{c}{$T=500$} & \multicolumn{1}{c}{$T=1500$} \\
\hline & & & \\
Panel A: $k=5$ & & \\
\hline$d$ & 0.60 & $0.591(0.024)$ & $0.601(0.015)$ \\
$\beta$ & -0.10 & $-0.109(0.052)$ & $-0.106(0.040)$ \\
$c$ & 0.05 & $0.059(0.010)$ & $0.058(0.008)$ \\
$\nu_{0}$ & 9.00 & $9.204(1.287)$ & $9.080(0.776)$ \\
$\nu_{1}$ & 50.00 & $49.982(3.340)$ & $50.228(2.298)$ \\
$\nu_{2}$ & 35.00 & $35.235(1.636)$ & $34.908(1.260)$ \\
\hline & & & \\
Panel B: & $k=15$ & & \\
\hline$d$ & 0.60 & $0.587(0.009)$ & $0.598(0.006)$ \\
$\beta$ & 0.15 & $0.136(0.014)$ & $0.147(0.008)$ \\
$c$ & 0.05 & $0.059(0.003)$ & $0.059(0.003)$ \\
$\nu_{0}$ & 12.00 & $12.136(1.089)$ & $12.069(0.653)$ \\
$\nu_{1}$ & 70.00 & $69.769(1.173)$ & $69.859(0.675)$ \\
$\nu_{2}$ & 60.00 & $60.249(0.843)$ & $60.114(0.484)$ \\
\hline
\end{tabular}

\section{Simulation Experiment}

Before presenting the empirical results, we perform a Monte Carlo study to investigate the statistical properties of the maximum likelihood estimator for $\theta$. To that end, we simulate $T$ time series observations of $k \times 1$ daily returns and $k \times k$ daily realized covariance matrices using the FIGAS tF model as the true data genarating process (DGP). We set $T$ equal to 500 and 1500 respectively, and choose $k$ equal to 5 and 15 . The chosen parameters are based on the FIGAS $(1, d, 0)$ model estimated in the application in Section 4 . For $k=5$, we choose $\beta=-0.10, \nu_{0}=9, \nu_{1}=50, \nu_{2}=35, d=0.60$, and $\tilde{\Omega}=c I_{k}$ with $c=0.05$. For $k=15$, we set $\beta=0.15, \nu_{0}=12, \nu_{1}=70, \nu_{2}=60$, while the other parameters remain the same. For each simulated series, we estimate $\theta$ by numerically maximizing the likelihood function.

Table 1 presents the results based on 1000 replications. Clearly, all parameters are estimated near their true values. The standard deviations decrease if the sample size $T$ increases or if the cross-dimensional dimension $k$ becomes larger. There is a slight upward bias in $c$, which is probably due to a long lasting effect of the initialization of the initial covariance matrices to their unconditional expectation. This bias tapers off (not shown) if the sample size grows substantially larger. We also observe that the fractional integration 
Table 2: S\&P 500 constituents

This table lists 15 companies listed at the S\&P 500 index during the period January 2, 2001 until December 31, 2012. Ts denotes the Ticker Symbol and PERMNO is the CRSP identifier.

\begin{tabular}{llcll}
\hline Nr. & Ts & Permno & Name & Subsector \\
\hline 1 & AA & 24643 & Alcoa Inc. & Materials \\
2 & AXP & 59176 & American Express Company & Financials \\
3 & BA & 19561 & The Boeing Company & Industrials \\
4 & CAT & 18542 & Caterpillar Inc. & Industrials \\
5 & GE & 12060 & General Electric Company & Industrials \\
6 & HD & 66181 & The Home Depot & Consumer discretionary \\
7 & HON & 10145 & Honeywell International & Industrials \\
8 & IBM & 12490 & International Business Machines & IT \\
9 & JPM & 47896 & JP Morgan & Financials \\
10 & KO & 11308 & Coca-Cola & Consumer staples \\
11 & MCD & 43449 & McDonald's & Consumer discretionary \\
12 & PFE & 21936 & Pfizer & Health care \\
13 & PG & 18163 & Procter \& Gamble & Consumer staples \\
14 & WMT & 55976 & Wal-Mart Stores Inc. & Consumer staples \\
15 & XOM & 11850 & Exxon Mobil & Energy \\
\hline
\end{tabular}

parameter $d$ is estimated accurately.

\section{Empirical Application}

In this section we apply the FIGAS model to an empirical data set of 15 US equities. Our aim is to describe the covariance dynamics both in-sample and out-of-sample. All equities are part of the S\&P 500 index. We first provide some of the stylized facts of the data. Next, we introduce our competing benchmark models. Finally, we test the in-sample and out-of-sample performance of the different models.

\subsection{Data}

The data consist of daily returns and daily realized covariances measures for 15 US equities. Table 2 provides an overview of the companies considered in our data set. The data spans the period January 2, 2001 until December 31, 2012 and contains $T=3017$ trading days. We observe consolidated trades (transaction prices) extracted from the Trade and Quote (TAQ) database from 9:30 until 16:00 with a time-stamp precision of one second. We first clean the high-frequency data following the guidelines of Barndorff-Nielsen et al. (2009) and 

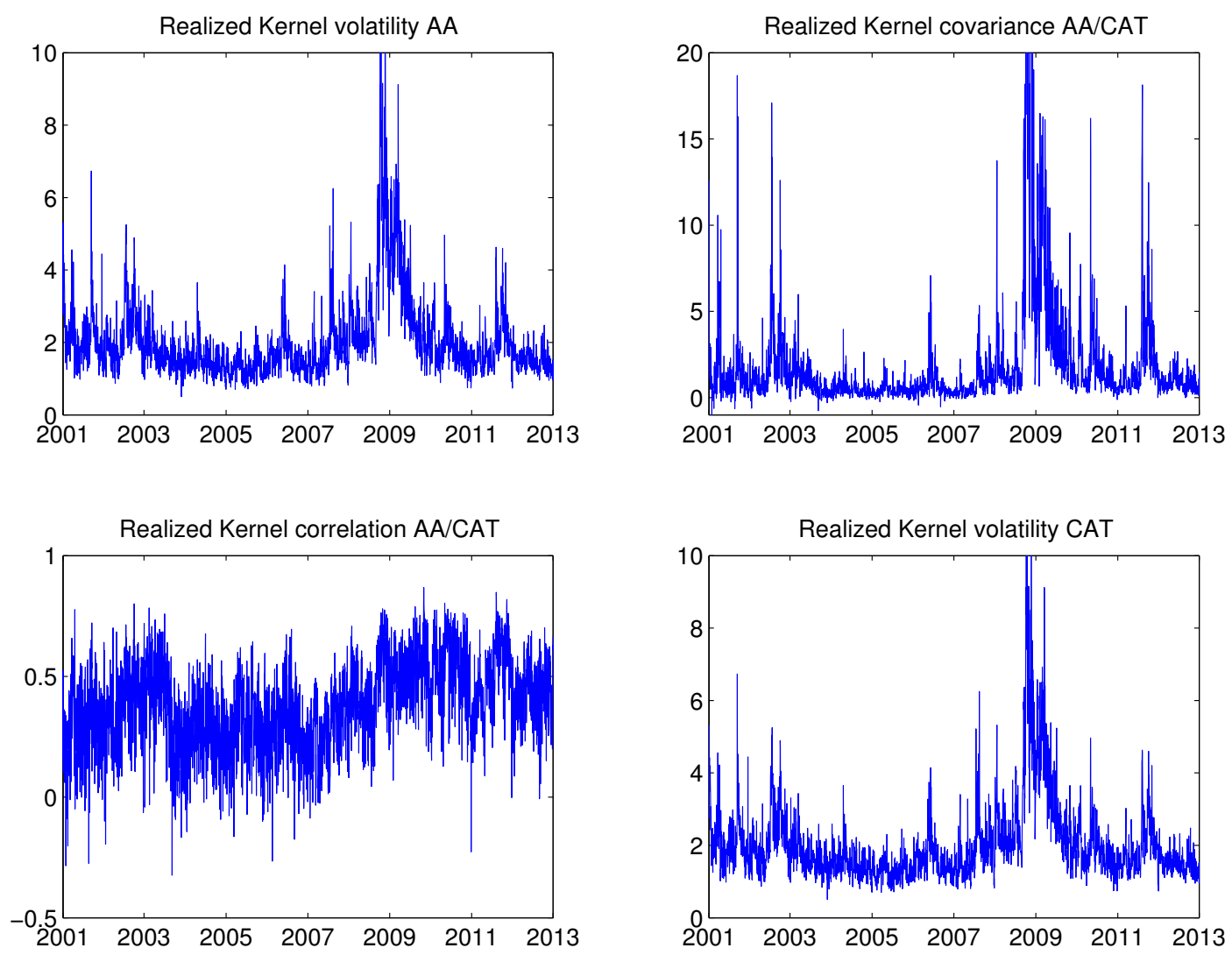

Figure 1: Realized Kernel estimates of AA/CAT

This figure shows daily realized kernel volatilities (square root of the variance) of Alcoa Inc. (AA) and Caterpillar Inc. (CAT) returns on the diagonal panels. The off-diagonal panels contain the realized kernel covariance (upper-right) and correlation (lower-left) between the two asset returns. The sample spans the period from January 2, 2001 until December 31, 2012 ( $T=3017$ days).

Brownlees and Gallo (2006). ${ }^{2}$ Next, we construct realized kernels using the refresh-timesampling methods of Barndorff-Nielsen et al. (2011) with the same hyper-parameters as used by Hansen et al. (2014).

Figure 1 shows a snapshot of the data by plotting the realized variances (based on the kernel approach) of Alcoa Inc. (AA) and Caterpillar Inc. (CAT) in the diagonal panels, and the realized correlation and covariance in the off-diagonal panels. The figure shows that both the realized (co)variance(s) and the realized correlation contain a substantial number of spikes. The spikes do not only occur during the global financial crisis, but also during other periods such as the early 2000s. This motivates the use of our FIGAS tF framework based on the fat-tailed matrix- $F$ and Student's $t$ distributions, which automatically downweights the impact of such incidental observations on the volatility and covariance dynamics.

\footnotetext{
${ }^{2}$ See Web Appendix A for more details.
} 

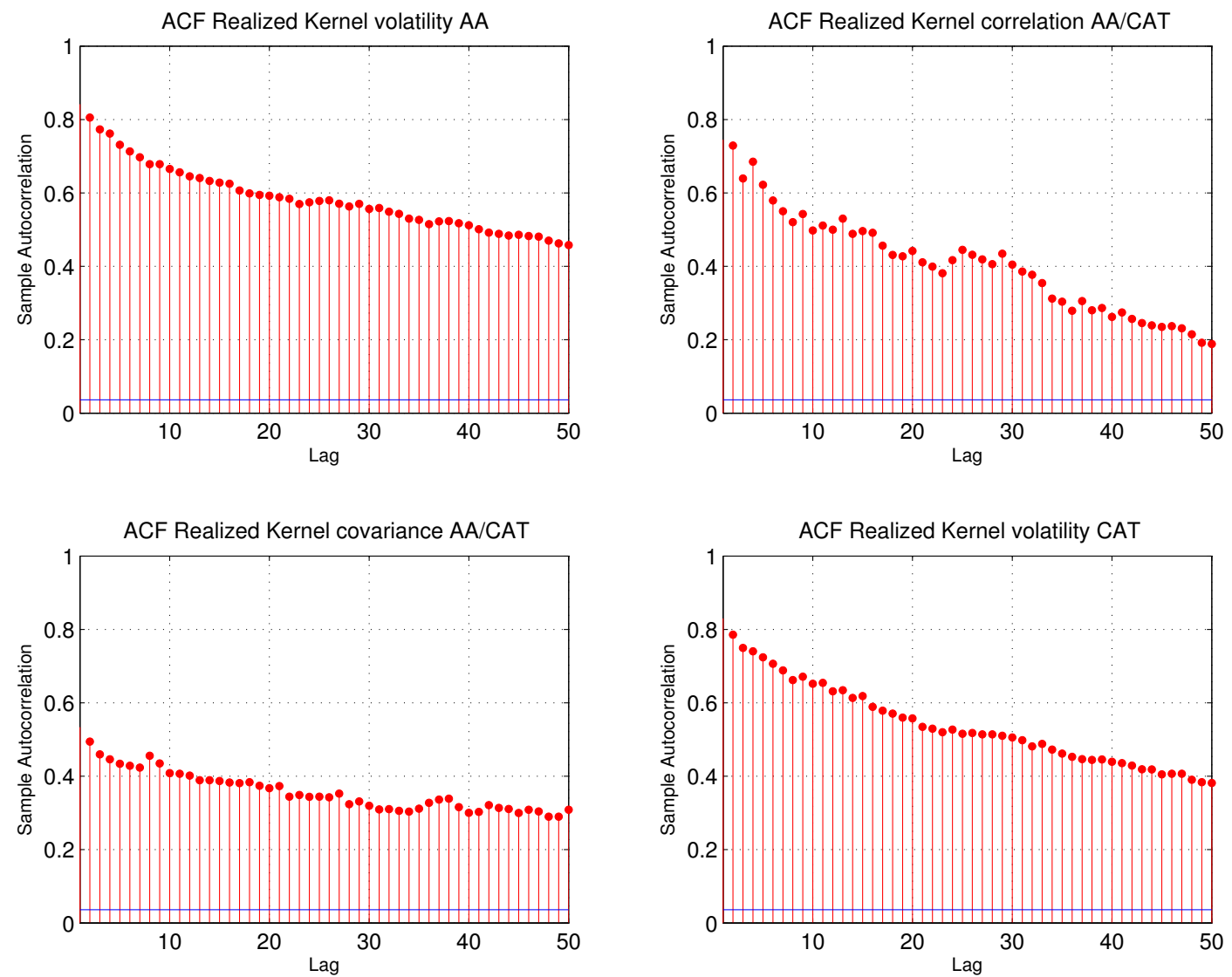

Figure 2: Empirical autocorrelation functions of realized kernels

This figure shows the autocorrelation function (ACF) for lag 1 until 50 of daily realized kernel volatilities (square root of variance) of Alcoa Inc. (AA) and Caterpillar Inc. (CAT) in the diagonal panels. The offdiagonal panels contain the ACF of the realized kernel covariance (upper-right) and correlation (lower-left) between the two asset returns. The sample is January 2, 2001 until December 31, 2012 ( $T=3017$ days).

The autocorrelation functions in Figure 2 strongly suggest that the realized covariance matrix displays long-memory behavior. After lag 50, the autocorrelation is around 0.4 for the realized kernel volatilities of AA and CAT. Likewise, the autocorrelation of the realized covariance and correlation is equal to 0.25 and 0.3 at this long lag length. This provides an empirical motivation to incorporate long-memory features into the model.

\subsection{Alternative forecasting models}

To benchmark the performance of our FIGAS tF model, we use three relevant alternative models: the multivariate extension of the HAR model (Corsi, 2009), put forward by Chiriac and Voev (2011), the multivariate HEAVY model of Noureldin et al. (2012) and the short-memory GAS tF model of Opschoor et al. (2017). As a fourth benchmark, we also considered the long-memory extension of the RiskMetrics model (Zumbach, 2006). This 
model, however, appears to be inferior compared to all other models considered. Results are therefore relegated to Web Appendix B.

Our first benchmark does not directly model $R K_{t}$, but first computes the matrix' choleski decomposition $R K_{t}=P_{t} P_{t}^{\prime}$, where $P_{t}$ is lower triangular. The multivariate HAR model then considers $X_{t}=\operatorname{vech} P_{t}$ as a function of lagged daily, weekly and monthly (transformed) volatilities, where vech $P_{t}$ stacks the lower triangular elements of $P_{t}$ into a vector:

$$
X_{t+1}=\alpha+\beta_{1} X_{t}+\beta_{2} X_{t}^{w}+\beta_{3} X_{t}^{m}+u_{t+1},
$$

where $X_{t}^{w}$ and $X_{t}^{m}$ are defined as $N^{-1} \sum_{i=0}^{N-1} X_{t-i}$ with $N=5$ (weekly) and 22 (monthly) respectively. Finally, $\alpha$ represents a $k(k+1) / 2$ vector of coefficients and $\beta_{j}(j=1,2,3)$ are scalar parameters. All parameters are estimated by OLS.

The multivariate HEAVY model incorporates realized measures into the volatility specification by proposing a system of two multivariate GARCH equations for the quantities $V_{t}=\mathbb{E}_{t}\left[y_{t} y_{t}^{\prime} \mid \mathcal{F}_{t-1}\right]$ and $M_{t}=\mathbb{E}_{t}\left[R K_{t} \mid \mathcal{F}_{t-1}\right]$. The innovations in both of these equations are the realized (co)variance measures as gathered in the matrix $R K_{t}$. The dynamics are given by

$$
\begin{aligned}
V_{t+1} & =C_{V} C_{V}^{\prime}+\alpha_{V} R K_{t}+\beta_{V} V_{t} \\
M_{t+1} & =C_{M} C_{M}^{\prime}+\alpha_{M} R K_{t}+\beta_{M} M_{t}
\end{aligned}
$$

where $\alpha_{V}, \alpha_{M}, \beta_{V}$, and $\beta_{M}$ are scalar parameters, and $C_{V}$ and $C_{M}$ are lower triangular matrices. The scalar parameters of both equations are estimated separately by maximum likelihood, assuming a singular Wishart distribution for $y_{t} y_{t}^{\prime}$ and a standardized Wishart distribution with $k$ degrees of freedom for $R K_{t}$. The matrices $C_{V}$ and $C_{M}$ are typically estimated by covariance targeting, as discussed by Noureldin et al. (2012). We follow this approach when implementing the model in the remaining analysis.

Our final benchmark is the GAS tF model, which is the short memory equivalent of our FIGAS model. All benchmark models allow for easy $h$-step ahead prediction of $V_{t}$. In case of the HEAVY model, the second transition equation delivers forecasts of $R K_{t+h}$ for $h=1,2, \ldots$, which can subsequently be inserted into the first equation to obtain $V_{t+h}$. The 
$h$-step ahead forecast of $V_{t}$ of the FIGAS model follows directly from (8): $V_{t+h}$ depends on $s_{t+h-1}^{*}, s_{t+h-2}^{*}, \ldots s_{t}^{*}, s_{t-1}^{*}, \ldots$, with $s_{t}^{*}=s_{t}+V_{t}$ by definition. Given the property that $\mathbb{E}_{t}\left[s_{t+h} \mid \mathcal{F}_{t}\right]=0_{k}$ for any value of $h \geq 1, V_{t+h}$ is obtained recursively by setting the values of future score matrices $s_{t}$ to zero. Similar results hold for the GAS tF model.

We follow Bollerslev et al. (2016) by considering direct forecasts in case of the the multivariate HAR model. These forecasts are obtained by running the following regression:

$$
X_{t+h}=\alpha+\beta_{1} X_{t}+\beta_{2} X_{t}^{w}+\beta_{3} X_{t}^{m}+u_{t+h},
$$

where $h$ stands for the forecast horizon. As indicated by Bollerslev et al. (2016), direct forecasts might be more adequate than iterative forecasts due to the possibility of model misspecification.

\subsection{Model Evaluation Procedure}

We follow Noureldin et al. (2012) and compare the in-sample and out-of-sample statistical fit of the models by computing the quasi-likelihood loss function:

$$
Q L I K_{t, h}\left(R K_{t+h}, V_{t+h \mid t}^{a}\right)=\log \left|V_{t+h \mid t}^{a}\right|+\operatorname{tr}\left(\left(V_{t+h \mid t}^{a}\right)^{-1} R K_{t+h}\right)
$$

with $V_{t+h \mid t}^{a}$ the covariance matrix forecast for time $t+h$ given all information up to time $t$ based on model $a$. Note that we use $R K_{t+h}$ as a proxy of the true covariance matrix. Insample, $h$ is set to zero. Since $V_{t}$ is known at time $t-1$, the criteria can also be interpreted as one-step ahead forecasting criteria. As indicated by Patton (2011), the QLIK loss-function implies a consistent ranking of volatility models since it is robust to noise in the proxy $R K_{t}$. To assess the in-sample performance, we set $h=0$ and note that $V_{t}$ only depends on the information in $\mathcal{F}_{t-1}$ for all three models considered. For the out-of-sample performance, we set $h>0$.

We additionally test the predictive performance of the models using the framework of Giacomini and White (2006). We start by computing the difference in loss functions between 
two competing models $a$ and $b$,

$$
d_{t, h}(a, b)=Q L I K_{t, h}\left(R K_{t+h}, V_{t+h \mid t}^{a}\right)-Q L I K_{t, h}\left(R K_{t+h}, V_{t+h \mid t}^{b}\right)
$$

for $t=R+1, \ldots T-h$, where the parameters are estimated based on a rolling window of $T_{w}=1500$ observations. The difference $d_{t}$ can be interpreted as a difference between two Kullback-Leibler (KL) divergences. Even if the underlying two models are both misspecified, the difference in their KL divergences still provides a valid assessment criterion. The corresponding null-hypothesis of equal predictive ability is given by $H_{0}: \mathbb{E}\left[d_{t, h}(a, b)\right]=0$ for all $T-h-R$ out-of-sample forecasts, which can be tested using the Diebold and Mariano (1995) (DM) test-statistic

$$
D M_{h}(a, b)=\frac{\bar{d}_{h}}{\sqrt{\hat{s}_{h}^{2} /(T-h-R)}},
$$

with $\bar{d}_{h}$ the out-of-sample average of the loss differences, and $\hat{s}_{h}^{2}$ a HAC-consistent variance estimator of $d_{t, h}(a, b)$. A significantly negative value of $D M_{h}(a, b)$ means that model $a$ has a superior forecast performance over model $b$. The QLIK test can be used in-sample (interpreted as a 'one-step-ahead prediction') and out-of-sample. In the out-of-sample test, we choose $h=1,5,10$ and 22. In addition, we consider the cumulative forecasts $V_{t: t+N \mid t}=$ $\sum_{i=1}^{N} V_{t+i \mid t}$, where $N$ equals 5,10 and 22 , respectively.

As the above evaluation criteria are statistical in nature, we finally also assess the forecasting performance from a economic point of view. Motivated by the mean-variance optimization setting of Markowitz (1952), we do so by considering global minimum variance portfolios (GMVP); see for example Chiriac and Voev (2011); Engle and Kelly (2012), among others, who perform a similar analysis. The best forecasting model should provide portfolios with the lowest ex-post variance. Assuming that the investor's aim is to minimize the $h$-step portfolio volatility at time $t$ subject to a fully invested portfolio, the resulting GMVP weights $w_{t+h \mid t}$ are obtained by the solution of the quadratic programming problem

$$
\min w_{t+h \mid t}^{\prime} V_{t+h \mid t} w_{t+h \mid t}, \quad \text { s.t. } w_{t+h \mid t}^{\prime} \iota=1 \text {. }
$$

with $\iota$ a $k \times 1$ vector of ones. Similar as Chiriac and Voev (2011), we assess the predictive abil- 
ity of the different models by comparing the results to the ex-post realizations or 'oracle forecasts' of the conditional standard deviation, which are given by $\sigma_{p, t}=\sqrt{w_{t+h \mid t}^{\prime} R K_{t+h} w_{t+h \mid t}}$. We again test for significantly different portfolio standard deviations by means of the DM test statistic.

\subsection{In-sample results}

Table 3 shows parameter estimates and standard errors based on the sandwich (robust covariance matrix) estimator $S_{0}=A_{0}^{-1} B_{0} A_{0}^{-1}$ with $B_{0}$ the inverse Hessian of the likelihood evaluated at the optimum (information matrix), and $A_{0}$ the expected value of the outer product of the gradients at the optimum. We show the results for a selection of $k=5$ randomly chosen stocks and for the complete set of all 15 equities. 
Table 3: Parameter estimates, likelihoods and loss function

This table reports maximum likelihood parameter estimates of the FIGAS tF, GAS tF, HEAVY and the HAR model, applied to daily equity returns and daily realized kernels of 5 and 15 assets. In case of the HAR model (see (17)), we suppress the constant vector and report only $\beta_{1}$, $\beta_{2}$ and $\beta_{3}$. Asset identifiers are explained in Table 2. Standard errors are provided in parenthesis and based on the (sandwich) robust covariance matrix estimator. The $\alpha$ column corresponds to $\alpha$ coefficient of the GAS tF model (see (8)) and the $\alpha_{V}$ coefficient of the HEAVY model (see (18)) respectively. Likewise, $\beta$ corresponds with $\beta$ of the (FI)GAS tF model, the $\beta_{V}$ coefficient of the HEAVY model or the $\beta_{1}$ coefficient of the HAR model. The table reports the total log-likelihood, the BIC criteria as well as the mean of the QLIK loss function, which is defined in (21). For comparative reasons, the total likelihood consists of the sum of a Multivariate Normal and the Wishart distribution for the HEAVY and HAR models. The sample is January 2, 2001, until December 31, 2012 (3017 observations).

\begin{tabular}{|c|c|c|c|c|c|c|c|c|c|c|c|}
\hline \multicolumn{12}{|c|}{ Panel A: AA/BA/CAT/GE/KO } \\
\hline & $d$ & $\alpha$ & $\beta$ & $\beta_{2}$ & $\beta_{3}$ & $\nu_{0}$ & $\nu_{1}$ & $\nu_{2}$ & $\mathcal{L}^{*}$ & $\mathrm{BIC}$ & QLIK \\
\hline FIGAS & $\begin{array}{c}0.655 \\
(0.014)\end{array}$ & & $\begin{array}{l}-0.063 \\
(0.044)\end{array}$ & & & $\begin{array}{c}10.89 \\
(0.830)\end{array}$ & $\begin{array}{c}46.31 \\
(2.032)\end{array}$ & $\begin{array}{c}36.68 \\
(1.437)\end{array}$ & $-44,213$ & 88,466 & 7.645 \\
\hline GAS & & $\begin{array}{c}0.611 \\
(0.025)\end{array}$ & $\begin{array}{c}0.987 \\
(0.001)\end{array}$ & & & $\begin{array}{c}10.46 \\
(0.730)\end{array}$ & $\begin{array}{c}46.39 \\
(1.669)\end{array}$ & $\begin{array}{c}35.58 \\
(1.112)\end{array}$ & $-44,686$ & 89,412 & 7.664 \\
\hline HEAVY & & $\begin{array}{c}0.284 \\
(0.049)\end{array}$ & $\begin{array}{c}0.589 \\
(0.080)\end{array}$ & & & & & & $-71,090$ & 142,211 & 7.894 \\
\hline HAR & & & $\begin{array}{c}0.306 \\
(0.016)\end{array}$ & $\begin{array}{c}0.418 \\
(0.023)\end{array}$ & $\begin{array}{c}0.214 \\
(0.020)\end{array}$ & & & & $-69,456$ & 139,056 & 7.684 \\
\hline
\end{tabular}

Panel B: AA/AXP/BA/CAT/GE/HD/HON/IBM/JPM/KO/MCD/PFE/PG/WMT/XOM

\begin{tabular}{|c|c|c|c|c|c|c|c|c|c|c|c|}
\hline & $d$ & $\alpha$ & $\beta$ & $\beta_{2}$ & $\beta_{3}$ & $\nu_{0}$ & $\nu_{1}$ & $\nu_{2}$ & $\mathcal{L}^{*}$ & $\mathrm{BIC}$ & QLIK \\
\hline FIGAS & $\begin{array}{c}0.647 \\
(0.009)\end{array}$ & & $\begin{array}{c}0.166 \\
(0.015)\end{array}$ & & & $\begin{array}{c}12.79 \\
(0.612)\end{array}$ & $\begin{array}{c}67.17 \\
(1.218)\end{array}$ & $\begin{array}{c}62.18 \\
(0.989)\end{array}$ & 5,486 & $-10,931$ & 18.88 \\
\hline GAS & & $\begin{array}{c}0.386 \\
(0.012)\end{array}$ & $\begin{array}{c}0.991 \\
(0.001)\end{array}$ & & & $\begin{array}{c}12.30 \\
(0.571)\end{array}$ & $\begin{array}{c}66.63 \\
(1.161)\end{array}$ & $\begin{array}{c}61.54 \\
(0.975)\end{array}$ & 3,092 & $-6,143$ & 18.96 \\
\hline HEAVY & & $\begin{array}{c}0.160 \\
(0.019)\end{array}$ & $\begin{array}{c}0.738 \\
(0.033)\end{array}$ & & & & & & $-132,489$ & 265,011 & 20.06 \\
\hline HAR & & & $\begin{array}{c}0.204 \\
(0.008) \\
\end{array}$ & $\begin{array}{c}0.419 \\
(0.012) \\
\end{array}$ & $\begin{array}{c}0.296 \\
(0.011) \\
\end{array}$ & & & & $-118,435$ & 237,854 & 19.45 \\
\hline
\end{tabular}


The results in Table 3 show that the FIGAS tF model has the best fit to the data compared to the other models. In a preliminary analysis, the coefficient $\phi$ in the FIGAS specification turned out to be statistically insignificant, such that we estimate a $\operatorname{FIGAS}(1, d, 0)$ model as our preferred fractionally integrated score-driven model.

Based on the QLIK loss function, the FIGAS tF has the best value, followed by the GAS tF, HAR and the HEAVY model, respectively. Comparing the FIGAS, GAS, and HEAVY models, the QLIK values suggest that the largest gain is obtained by introducing the scoredriven dynamics: the average QLIK drops from 7.89 (20.06) for the HEAVY model to 7.66 (18.96) for the GAS model. Hence allowing for fat-tailedness in both the return observations and realized covariance kernels improves the fit substantially. The further drop in QLIK when moving from GAS to FIGAS is more modest, but still sizable given the sample size.

For $k=5$, the simple HAR model still does quite well. The HAR model's relative performance, however, quickly deteriorates in higher dimensions such as $k=15$, as is seen in Panel B of Table 3.

The likelihood and the BIC values for the different models underline that the FIGAS tF model provides a better fit to the data than the GAS and HEAVY models. Especially when $k=15$ the differences are large. In order to compare the FIGAS type model with the HEAVY and HAR models, we compute the total likelihood of these models as the sum of a Multivariate Normal (for the returns) and a Wishart distribution with $k$ degrees of freedom for the realized kernels. This makes the likelihood comparable to that of the FIGAS model, as the latter is the sum of a multivariate Student's $t$ and a matrix- $F$ distribution.

We see a clear distinction between the likelihoods of the HEAVY and HAR model and the FIGAS model. This occurs mainly due to the likelihood contribution for the realized kernels. More specifically, this contribution equals 25,000 points $(k=5)$ or 120,000 points $(k=15)$ when going from the Wishart distribution (HEAVY and HAR model) to the matrix$F$ distribution (FIGAS model). Fat-tailedness of $R K_{t}$ thus appears a prevalent feature in the data.

The likelihood values for the short-memory GAS specification and the FIGAS model are easier to compare. The likelihood increases by almost 450 points $(k=5)$ or even more than 2300 points $(k=15)$ for the same number of parameters. This underlines that the long-memory features also play an important role in explaining the volatility and correlation 
(A)

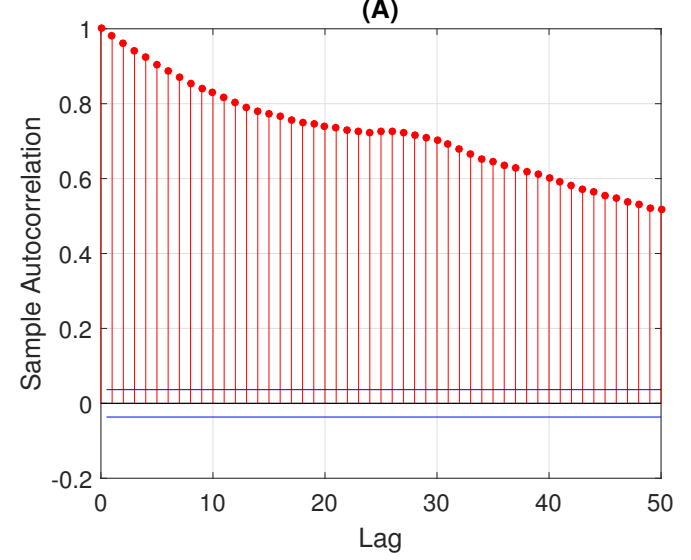

(B)

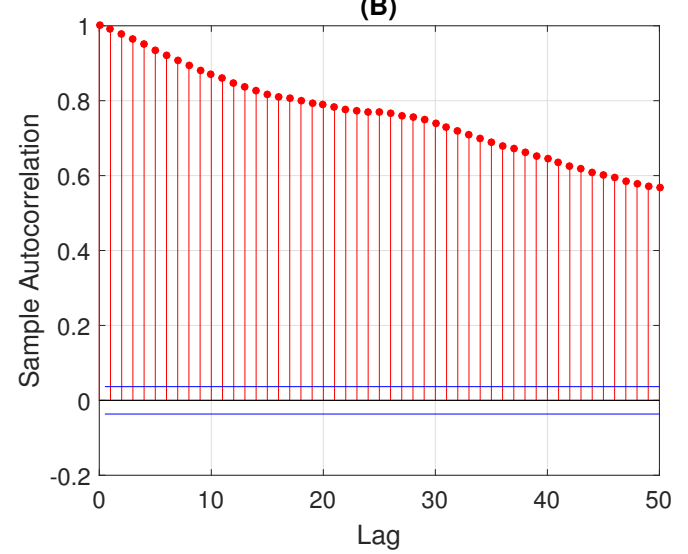

Figure 3: FIGAS implied correllograms for $V_{t}$ for $k=5$ (left) and $k=15$ (right) This figure plots the implied correllograms of the conditional variance of Alcoa Inc. (AA) corresponding with the FIGAS tF model based on parameter estimates in Table 3. The left panel shows the correllogram implied by the estimated model on $\mathrm{AA} / \mathrm{BA} / \mathrm{CAT} / \mathrm{GE} / \mathrm{KO}$, while the right panel uses all $(k=15)$ all equities.

dynamics.

Looking at the individual parameter estimates, we first note the positive and significant long-memory coefficient $d$. A similar estimate of $d$ is found by for example Proietti (2016) in the univariate case. The value of $d$ is highly robust across the dimensions considered and indicates that autocorrelations only die out very slowly.

The high degree of persistence in the FIGAS tF model is mirrored by the other models. For example, for the HAR model $\beta_{1}+\beta_{2}+\beta_{3}$ is also close to 1 . Similarly, the estimate of the autoregressive coefficient for the short-memory GAS model is very close to $1(\beta \approx 0.99)$, indicating a strong persistence. The value of $\beta$ of the FIGAS tF model changes from insignificantly negative for $k=5$ to significantly positive for the case of all equities. Based on Proposition (1), we empirically satisfy the constraint for positive definiteness of the resulting covariance matrices $V_{t}$ for both dimensions. Though the value of $B$ is negative for $k=5$ and positive for $k=15$, both values imply a highly similar set of autocovariance functions; see Figure 3. If anything, the increase of the dimension leads to a slightly stronger long-memory feature.

The degrees of freedom parameter $\nu_{2}$ is estimated at around 35 and 65 for 5 and 15 dimensions, respectively. Despite that the value of $\hat{\nu_{2}}$ may appear high, such values already result in a substantial moderation of the effect of incidentally large observations $R K_{t}$ in (14) through the matrix weighting scheme. Also fat-tailedness for these values of $\nu_{2}$ is considerably larger than that of the Wishart distribution (see also Opschoor et al., 2017) 

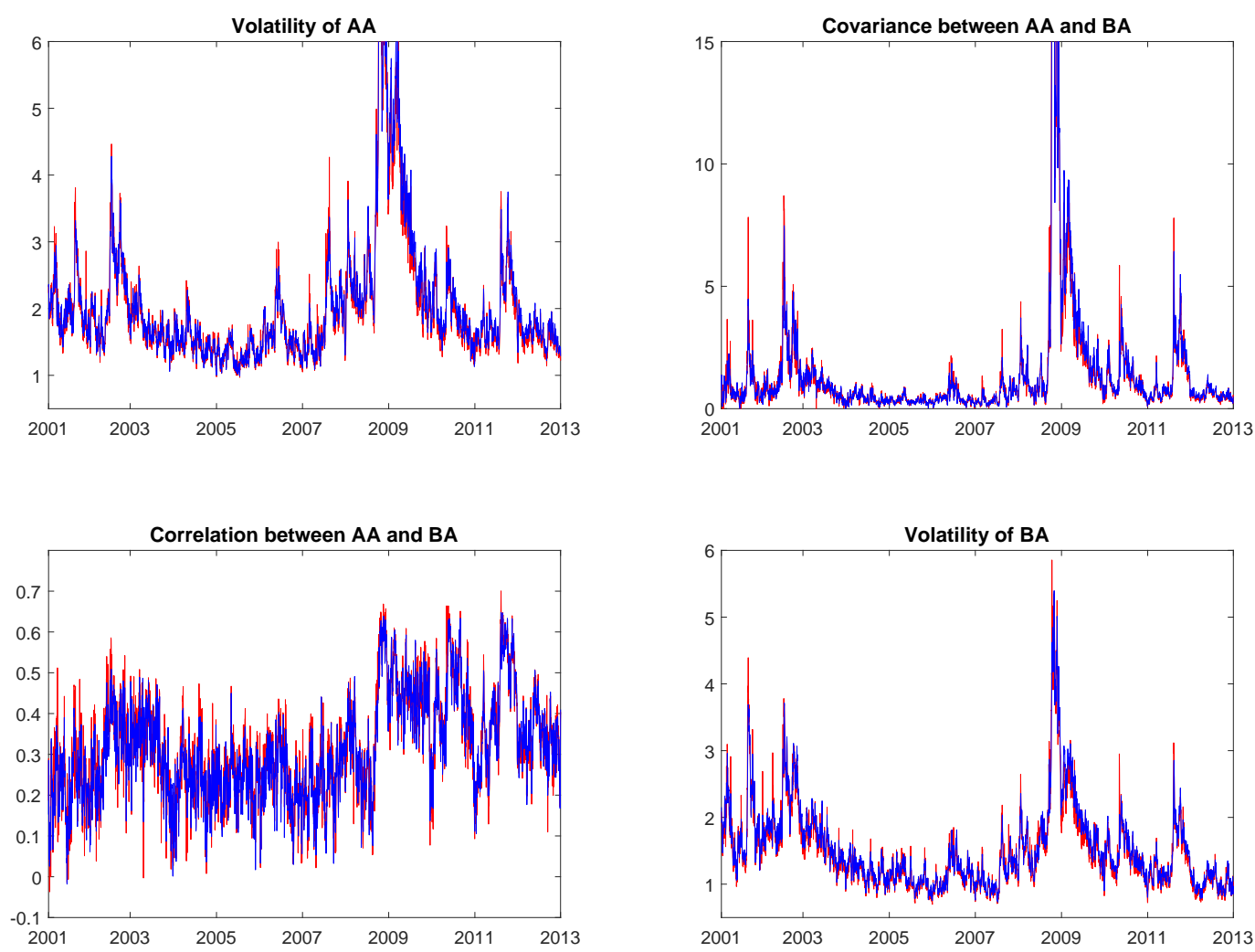

Figure 4: Estimated volatilities and correlations

This figure plots the estimated volatilities of AA and BA (see Table 2) in the upper-left and lower-right panels, and the pairwise covariances and correlations in the upper-right and lower-left panels respectively. Time varying parameter paths are estimated using the FIGAS tF model (blue line) and HAR model (red line). The estimates are based on the full sample, January 2, 2001 until December 31, 2012 (3017 observations).

Figure 4 plots some of the fitted volatilities and correlations. We show the results for Alcoa (AA) and Boeing (BA) for the FIGAS tF model (blue line) and the HAR model of equation (17) (red line). The figure shows remarkable differences between the two models for both the volatility and the covariances and correlations. Focusing first on the volatilities and covariances, the robust transition scheme based on the Student's $t$ and matrix- $F$ score dynamics produces considerably fewer spikes: red (HAR) spikes stand out much more clearly than the blue (FIGAS) ones. Notable differences are apparent for both companies during the periods 2001-2003, 2007-2008, and 2010-2011. The FIGAS framework is able to mitigate the impact of temporary $R K_{t}$ and $y_{t} y_{t}^{\prime}$ observations on the estimates of $V_{t}$.

We conclude that already for short horizons there is evidence that a combination of long-memory and fat-tailedness improves model performance. We expect the long-memory properties to become even more important once we move into longer forecasting horizons, as we do next. 


\subsection{Out-of-sample results}

In our out-of-sample analysis, we assess both the short-term and long-term forecasting performance of the FIGAS tF model. We consider $h$-step ahead forecasts, with $h=1,5,10$, and 22. In addition, we consider aggregated covariance forecasts for the next one or two trading weeks and for the next month, i.e. $V_{t: t+h}=V_{t+1}+V_{t+2}+\ldots+V_{t+h}$ with $h=5,10,22$. Similar to the in-sample analysis of the previous subsection, we compare the FIGAS tF model with the HEAVY model, the GAS tF model and the multivariate HAR model.

We test the predictive ability of the different models based on the loss-differences of the QLIK loss function (21) using the test-statistic defined in (23). We use a moving window of 1500 observations and re-estimate the parameters after each 25 observations ( $\approx$ one month). The first in-sample period corresponds to the period January 2001 until December 2006, which is well before the financial crisis of October 2008. This current forecasting experiment therefore constitutes a major robustness test for all the models considered.

Table 4 contains the results for the whole out-of-sample period (Panel A). We present the results for the 5 -dimensional case, as well as for all assets $(k=15)$. Negative $t$-test statistics (in parentheses) indicate that the FIGAS tF model performs better. The overall significant negative values in Panel A for horizons $h=1,5,10,22$ as well as for the aggregated forecasts clearly show that the FIGAS model statistically outperforms the HEAVY and HAR models. The short-memory GAS model still does quite well for horizons of (up to) $h=5$ or 10 , though worse than the FIGAS specification. Also here, however, the FIGAS model does significantly better for longer horizons such as $h=22$ and $h=1: 22$. The improvements due to the long memory features of the FIGAS specification thus appears particularly pronounced in cases where they matter most, namely at long horizon forecasting.

To further investigate where the performance of the fractionally integrated specification comes from, we split the sample in two periods: the Financial Crisis period (July 2007 - December 2009) and the Non-crisis period (Dec 2006 - July 2007 and January 2010 - December 2012). Panels B and C of Table 4 show that discriminating between the crisis and non-crisis periods provides us two additional remarkable insights. First, the differences between the average QLIK values between the FIGAS model and the HAR and HEAVY models increase during the crisis compared to the non-crises period. This holds especially for $k=15$. The 
Table 4: Test-statistics on predictive ability (QLIK criterion)

This table shows test statistics on superior predictive ability between the FIGAS tF model and the GAS tF, HEAVY or multivariate HAR model respectively, based on the QLIK loss function defined in (21). The test is based based on 1,5,10 and 22-step ahead predictions of the covariance matrix, applied to 5 and 15 (all) equities. Panel A present results for full out-of-sample (December 2006 - December 2012), Panel $\mathrm{B}$ describes the results for the Financial Crisis period (July 2007 - December 2009) $k=15$ and panel C shows the results for the Non-crisis period (Dec 2006 - July 2007 and January 2010 - December 2012). The subpanels 1 and 2 correspond to number of considered assets, i.e. $k=5$ and $k=15$. We report the average QLIK loss for each model with the associated DM-type of test statistic in parentheses. A negative test statistic indicates superior predictive ability of the FIGAS tF model. We use a moving window of 1500 observations. The prediction period contains 1495 observations.

\begin{tabular}{|c|c|c|c|c|c|c|c|}
\hline & 1 & 5 & 10 & 22 & $1: 5$ & $1: 10$ & $1: 22$ \\
\hline \multicolumn{8}{|c|}{ Panel A: Full out-of-sample } \\
\hline \multicolumn{8}{|c|}{ A.1: AA/BA/CAT/GE/KO } \\
\hline FIGAS & $\begin{array}{c}8.039 \\
(-)\end{array}$ & $\begin{array}{l}8.417 \\
(-)\end{array}$ & $\begin{array}{c}8.677 \\
(-)\end{array}$ & $\begin{array}{c}9.119 \\
(-)\end{array}$ & $\begin{array}{l}16.30 \\
(-)\end{array}$ & $\begin{array}{c}19.93 \\
(-)\end{array}$ & $\begin{array}{c}24.16 \\
(-)\end{array}$ \\
\hline GAS & $\begin{array}{l}8.057 \\
(-2.8)\end{array}$ & $\begin{array}{l}8.438 \\
(-0.9)\end{array}$ & $\begin{array}{l}8.754 \\
(-1.5)\end{array}$ & $\begin{array}{l}9.317 \\
(-1.9)\end{array}$ & $\begin{array}{l}16.32 \\
(-1.0)\end{array}$ & $\begin{array}{l}19.96 \\
(-1.2)\end{array}$ & $\begin{array}{l}24.24 \\
(-1.8)\end{array}$ \\
\hline HEAVY & $\begin{array}{c}8.259 \\
(-14.1)\end{array}$ & $\begin{array}{l}8.678 \\
(-7.7)\end{array}$ & $\begin{array}{l}9.009 \\
(-3.4)\end{array}$ & $\begin{array}{l}9.706 \\
(-2.2)\end{array}$ & $\begin{array}{c}16.54 \\
(-10.6)\end{array}$ & $\begin{array}{l}20.19 \\
(-6.1)\end{array}$ & $\begin{array}{l}24.50 \\
(-3.4)\end{array}$ \\
\hline HAR & $\begin{array}{l}8.061 \\
(-2.1)\end{array}$ & $\begin{array}{l}8.512 \\
(-3.1)\end{array}$ & $\begin{array}{l}8.836 \\
(-2.2)\end{array}$ & $\begin{array}{l}9.503 \\
(-1.6)\end{array}$ & $\begin{array}{c}19.73 \\
(-33.9)\end{array}$ & $\begin{array}{c}26.14 \\
(-34.5)\end{array}$ & $\begin{array}{c}33.77 \\
(-26.4)\end{array}$ \\
\hline
\end{tabular}

\begin{tabular}{lccccccc} 
A.2: All assets $(k=15)$ & & & & & \\
\hline FIGAS & 19.06 & 20.03 & 20.76 & 21.88 & 43.75 & 54.60 & 67.18 \\
& $(-)$ & $(-)$ & $(-)$ & $(-)$ & $(-)$ & $(-)$ & $(-)$ \\
GAS & 19.12 & 20.06 & 20.89 & 22.23 & 43.78 & 54.65 & 67.32 \\
& $(-3.2)$ & $(-0.5)$ & $(-1.0)$ & $(-1.8)$ & $(-0.9)$ & $(-0.8)$ & $(-1.3)$ \\
HEAVY & 20.61 & 21.64 & 22.49 & 23.98 & 45.32 & 56.21 & 68.90 \\
& $(-19.9)$ & $(-9.7)$ & $(-5.4)$ & $(-2.9)$ & $(-13.6)$ & $(-8.6)$ & $(-5.0)$ \\
HAR & 19.41 & 20.65 & 21.71 & 23.48 & 53.15 & 72.22 & 95.06 \\
& $(-6.8)$ & $(-5.1)$ & $(-3.1)$ & $(-1.9)$ & $(-32.1)$ & $(-34.0)$ & $(-27.4)$ \\
\hline
\end{tabular}


(continued from previous page)

\begin{tabular}{llllllll}
\hline 1 & 5 & 10 & 22 & $1: 5$ & $1: 10$ & $1: 22$ \\
\hline
\end{tabular}

Panel B: Crisis period

\begin{tabular}{lccccccc}
\hline B.1: AA/BA/CAT/GE/KO & & & & & \\
\hline FIGAS & 10.82 & 11.38 & 11.86 & 12.71 & 19.17 & 22.92 & 27.34 \\
& $(-)$ & $(-)$ & $(-)$ & $(-)$ & $(-)$ & $(-)$ & $(-)$ \\
GAS & 10.83 & 11.36 & 11.94 & 12.94 & 19.16 & 22.92 & 27.41 \\
& $(-0.6)$ & $(-0.5)$ & $(-0.7)$ & $(-1.0)$ & $(-0.6)$ & $(-0.1)$ & $(-0.7)$ \\
HEAVY & 11.04 & 11.72 & 12.42 & 13.83 & 19.44 & 23.27 & 27.92 \\
& $(-6.9)$ & $(-4.5)$ & $(-2.5)$ & $(-1.9)$ & $(-5.6)$ & $(-3.7)$ & $(-2.5)$ \\
HAR & 10.84 & 11.52 & 12.18 & 13.53 & 22.21 & 28.49 & 35.89 \\
& $(-1.1)$ & $(-2.3)$ & $(-2.0)$ & $(-1.5)$ & $(-16.2)$ & $(-15.7)$ & $(-11.3)$ \\
\hline \multirow{2}{*}{ B.2: All assets $(k=15)$} & & & & & \\
\hline FIGAS & 27.67 & 29.14 & 30.46 & 32.76 & 52.60 & 63.76 & 76.95 \\
& $(-)$ & $(-)$ & $(-)$ & $(-)$ & $(-)$ & $(-)$ & $(-)$ \\
GAS & 27.70 & 29.02 & 30.43 & 32.91 & 52.53 & 63.68 & 76.92 \\
& $(-0.7)$ & $(0.9)$ & $(0.1)$ & $(-0.4)$ & $(0.8)$ & $(0.5)$ & $(0.1)$ \\
HEAVY & 29.23 & 31.14 & 32.86 & 36.22 & 54.37 & 65.74 & 79.38 \\
& $(-9.4)$ & $(-5.2)$ & $(-3.2)$ & $(-2.1)$ & $(-6.8)$ & $(-4.6)$ & $(-3.1)$ \\
HAR & 28.14 & 30.16 & 32.25 & 36.12 & 60.72 & 79.37 & 101.5 \\
& $(-5.9)$ & $(-4.2)$ & $(-2.8)$ & $(-1.8)$ & $(-14.9)$ & $(-15.5)$ & $(-11.9)$ \\
\hline
\end{tabular}

Panel C: Non-Crisis period

\begin{tabular}{lccccccc}
\hline C.1: AA/BA/CAT/GE/KO & & & & & \\
\hline FIGAS & 6.00 & 6.25 & 6.35 & 6.49 & 14.21 & 17.75 & 21.82 \\
& $(-)$ & $(-)$ & $(-)$ & $(-)$ & $(-)$ & $(-)$ & $(-)$ \\
GAS & 6.03 & 6.30 & 6.42 & 6.67 & 14.24 & 17.79 & 21.91 \\
& $(-3.9)$ & $(-2.2)$ & $(-2.6)$ & $(-2.8)$ & $(-2.4)$ & $(-2.4)$ & $(-2.6)$ \\
HEAVY & 6.24 & 6.45 & 6.51 & 6.69 & 14.41 & 17.93 & 21.99 \\
& $(-15.8)$ & $(-10.3)$ & $(-5.4)$ & $(-3.3)$ & $(-13.2)$ & $(-9.4)$ & $(-5.3)$ \\
HAR & 6.02 & 6.31 & 6.39 & 6.56 & 17.92 & 24.41 & 32.22 \\
& $(-2.1)$ & $(-2.1)$ & $(-1.2)$ & $(-0.8)$ & $(-36.0)$ & $(-43.4)$ & $(-45.2)$ \\
\hline \multirow{2}{*}{ C.2: All assets $(k=15)$} & & & & & \\
\hline FIGAS & 12.76 & 13.35 & 13.67 & 13.92 & 37.28 & 47.89 & 60.03 \\
& $(-)$ & $(-)$ & $(-)$ & $(-)$ & $(-)$ & $(-)$ & $(-)$ \\
GAS & 12.85 & 13.50 & 13.92 & 14.41 & 37.38 & 48.04 & 60.30 \\
& $(-6.5)$ & $(-4.1)$ & $(-3.6)$ & $(-3.4)$ & $(-4.5)$ & $(-3.8)$ & $(-3.3)$ \\
HEAVY & 14.31 & 14.70 & 14.90 & 15.02 & 38.71 & 49.23 & 61.23 \\
& $(-29.4)$ & $(-20.7)$ & $(-15.3)$ & $(-9.4)$ & $(-25.4)$ & $(-19.7)$ & $(-14.0)$ \\
HAR & 13.03 & 13.69 & 13.99 & 14.23 & 47.62 & 66.99 & 90.32 \\
& $(-4.0)$ & $(-3.1)$ & $(-1.6)$ & $(-0.9)$ & $(-35.3)$ & $(-42.4)$ & $(-45.8)$ \\
\hline
\end{tabular}


crisis period is charactarized by more spikes, and the FIGAS specification can better deal with these due to the score dynamics and the fat-tailed distributional assumptions. The FIGAS model therefore performs particularly well in the crisis period compared to its HAR and HEAVY counterparts.

Second, the FIGAS model and the short-memory GAS specification perform similarly well during the crisis period, but not during the non-crisis period. In non-crisis years, the FIGAS model statistically outperforms its short-memory counterpart. Hence the fractionally integrated dynamics appear particularly valuable during calm periods, while accounting for fat-tailedness of returns and realized covariances is more important during turbulent years. To summarize, taking account of both fat-tailedness (during turbulent times) and long-memory effects (during calm periods) provides the FIGAS tF model with its superior forecasting performance over longer time spans.

Table 5 illustrates the economic significance of the covariance matrix forecasts by showing the mean of the ex-post conditional portfolio standard deviation, computed by implementing the period-by-period ex-ante minimum variance portfolio weights obtained from equation (24). Panels A and B display the average out-of-sample portfolio standard deviation and the associated DM test statistics vis-à-vis the FIGAS tF model (in parentheses). For all pairs of assets and all forecasting horizons considered, the FIGAS tF model produces the lowest ex-post portfolio standard deviation. This result also holds for the aggregated forecasts. The reductions in standard deviations are statistically significant compared to all of the benchmarks.

\section{Conclusions}

We introduced a new multivariate fractionally integrated model with score-driven volatility dynamics (FIGAS tF) that combines observed realized covariance matrices and vectorvalued return observations to estimate the dynamics of unobserved common covariance matrices. The proposed model explicitly acknowledges that realized (co)variances display long-memory behavior. It does so in a way that ensures positive definiteness of the covariance matrices by simple parameter restrictions in the model. In addition, the model takes into account that both realized covariance matrices and financial return data are typically 


\section{Table 5: Ex-post minimum variance portfolio standard-deviations}

This table shows results on a global minimum variance portfolio, based on 1, 5, 10 and 22-step ahead predictions of the covariance matrix, according to the FIGAS tF, GAS, HEAVY and the multivariate HAR model, applied to 5 and 15 equities. For each model, the table shows the ex-post mean of the daily portfolio volatility, whereas below the GAS, HEAVY and HAR model. The number between parentheses shows the test-statistic on equal portfolio volatility between the FIGAS tF model and the HEAVY, GAS or RM 2006 model. We use a moving window of 1500 observations. The prediction period runs from December, 2006 until December, 2012 (1495 observations).

\begin{tabular}{|c|c|c|c|c|c|c|c|}
\hline & 1 & 5 & 10 & 22 & $1: 5$ & $1: 10$ & $1: 22$ \\
\hline \multicolumn{8}{|c|}{ Panel A: AA/BA/CAT/GE/KO } \\
\hline FIGAS & $\begin{array}{c}0.925 \\
(-)\end{array}$ & $\begin{array}{c}0.933 \\
(-)\end{array}$ & $\begin{array}{c}0.938 \\
(-)\end{array}$ & $\begin{array}{c}0.946 \\
(-)\end{array}$ & $\begin{array}{c}2.121 \\
(-)\end{array}$ & $\begin{array}{c}3.032 \\
(-)\end{array}$ & $\begin{array}{l}4.567 \\
(-)\end{array}$ \\
\hline GAS & $\begin{array}{l}0.926 \\
(-5.6)\end{array}$ & $\begin{array}{l}0.937 \\
(-6.6)\end{array}$ & $\begin{array}{l}0.942 \\
(-4.2)\end{array}$ & $\begin{array}{l}0.952 \\
(-3.9)\end{array}$ & $\begin{array}{l}2.126 \\
(-6.5)\end{array}$ & $\begin{array}{l}3.043 \\
(-5.5)\end{array}$ & $\begin{array}{l}4.588 \\
(-4.5)\end{array}$ \\
\hline HEAVY & $\begin{array}{c}0.948 \\
(-21.1)\end{array}$ & $\begin{array}{c}0.954 \\
(-12.6)\end{array}$ & $\begin{array}{l}0.957 \\
(-9.3)\end{array}$ & $\begin{array}{l}0.961 \\
(-6.7)\end{array}$ & $\begin{array}{c}2.168 \\
(-14.6)\end{array}$ & $\begin{array}{c}3.096 \\
(-11.1)\end{array}$ & $\begin{array}{l}4.651 \\
(-8.2)\end{array}$ \\
\hline HAR & $\begin{array}{l}0.927 \\
(-3.6) \\
\end{array}$ & $\begin{array}{l}0.935 \\
(-3.2) \\
\end{array}$ & $\begin{array}{l}0.940 \\
(-2.8) \\
\end{array}$ & $\begin{array}{l}0.948 \\
(-2.2) \\
\end{array}$ & $\begin{array}{l}2.124 \\
(-4.2) \\
\end{array}$ & $\begin{array}{l}3.038 \\
(-3.8) \\
\end{array}$ & $\begin{array}{l}4.577 \\
(-3.3) \\
\end{array}$ \\
\hline \multicolumn{8}{|c|}{ Panel B: all equities } \\
\hline FIGAS & $\begin{array}{c}0.688 \\
(-)\end{array}$ & $\begin{array}{c}0.700 \\
(-)\end{array}$ & $\begin{array}{c}0.707 \\
(-)\end{array}$ & $\begin{array}{c}0.718 \\
(-)\end{array}$ & $\begin{array}{l}1.589 \\
(-)\end{array}$ & $\begin{array}{c}2.282 \\
(-)\end{array}$ & $\begin{array}{c}3.461 \\
(-)\end{array}$ \\
\hline GAS & $\begin{array}{l}0.689 \\
(-5.1)\end{array}$ & $\begin{array}{l}0.703 \\
(-5.4)\end{array}$ & $\begin{array}{l}0.711 \\
(-4.5)\end{array}$ & $\begin{array}{l}0.723 \\
(-4.1)\end{array}$ & $\begin{array}{l}1.593 \\
(-5.6)\end{array}$ & $\begin{array}{l}2.289 \\
(-5.1)\end{array}$ & $\begin{array}{l}3.477 \\
(-4.7)\end{array}$ \\
\hline HEAVY & $\begin{array}{c}0.737 \\
(-35.7)\end{array}$ & $\begin{array}{c}0.746 \\
(-21.9)\end{array}$ & $\begin{array}{c}0.750 \\
(-16.4)\end{array}$ & $\begin{array}{c}0.755 \\
(-12.3)\end{array}$ & $\begin{array}{c}1.693 \\
(-22.7)\end{array}$ & $\begin{array}{c}2.424 \\
(-17.1)\end{array}$ & $\begin{array}{r}3.655 \\
(-12.2)\end{array}$ \\
\hline HAR & $\begin{array}{l}0.693 \\
(-7.5)\end{array}$ & $\begin{array}{l}0.705 \\
(-5.7)\end{array}$ & $\begin{array}{l}0.712 \\
(-4.4)\end{array}$ & $\begin{array}{l}0.723 \\
(-3.7)\end{array}$ & $\begin{array}{l}1.597 \\
(-6.6)\end{array}$ & $\begin{array}{l}2.294 \\
(-5.3)\end{array}$ & $\begin{array}{l}3.480 \\
(-3.6)\end{array}$ \\
\hline
\end{tabular}

fat-tailed. The score-driven matrix-valued dynamics automatically correct for influential observations in either type of data. For S\&P500 equity returns over the period 2001-2012 we showed that both in-sample and out-of-sample and both statistically and economically the new model outperformed recent competitors such as the HEAVY model of Noureldin et al. (2012) and the multivariate HAR model of Corsi (2009) and Chiriac and Voev (2011). Interestingly, the fractionally integrated dynamics appear particularly valuable during calm periods. The outlier robust features of the model due to the score dynamics and the fat-tailed distributional assumptions, by contrast, are most useful during turbulent times. Combining the two, the FIGARCH model shows the best overall performance over the sample. 


\section{Acknowledgements}

We appreciate the comments of participants at the 69th European meeting of the Econometric Society (Geneva, August 2016), the Financial Econometrics and Empirical Asset Pricing Conference (Lancaster, July 2016), the 3rd annual meeting of the IAAE (Milan, June 2016), the 2015 NBER-NSF Time Series Conference (Vienna, September 2015), the 2nd International Workshop on Financial Markets and Nonlinear Dynamics (Paris, June 2015) and seminar participants at the Econometrics Brown Bag Seminar Series at Vrije Universiteit Amsterdam. Lucas and Opschoor thank the Dutch National Science Foundation (NWO, grant VICI453-09-005) for financial support.

\section{References}

Andersen, T., T. Bollerslev, F.X. Diebold and P. Labys (2001), The distribution of realized exchange rate volatility, Journal of the American Statistical Association 96, 42-55.

Asai, M., M. McAleer and J. Yu (2006), Multivariate stochastic volatility: a review, Econometric Reviews 25(2-3), 145-175.

Baillie, R.T., T. Bollerslev and H.O. Mikkelsen (1996), Forecasting multivariate realized stock market volatility, Journal of Econometrics 74, 3-50.

Barndorff-Nielsen, O.E. and N. Shephard (2002), Econometric analysis of realised volatility and its use in estimating stochastic volatility models, Journal of the Royal Statistical Society B 64, 253-280.

Barndorff-Nielsen, O.E., P.R. Hansen, A. Lunde and N. Shephard (2008), Designing realised kernels to measure the ex-post variation of equity prices in the presence of noise., Econometrica 76, 1481-1536.

Barndorff-Nielsen, O.E., P.R. Hansen, A. Lunde and N. Shephard (2009), Realized kernels in practice: trades and quotes, Econometrics Journal 12, 1-32.

Barndorff-Nielsen, O.E., P.R. Hansen, A. Lunde and N. Shephard (2011), Multivariate realized kernels: Consistent positive semi-definite estimators of the covariation of equity prices with noise and non-synchronous trading, Journal of Econometrics 12, 1-32.

Bauer, G.H. and K. Vorkink (2011), Forecasting multivariate realized stock market volatility, Journal of Econometrics 160, 93-101.

Blasques, C., S.J. Koopman and A. Lucas (2015), Information Theoretic Optimality of Observation Driven Time Series Models for Continuous Responses, 102(2), 325-343. 
Bollerslev, T. (1986), Generalized autoregressive conditional heteroskedasticity, Journal of Econometrics 31, 307-327.

Bollerslev, T., A.J. Patton and R. Quaedvlieg (2016), Modeling and forecasting (un) reliable realized covariances for more reliable financial decisions.

Bollerslev, T. and H.O. Mikkelsen (1996), Modeling and pricing long memory in stock market volatility, Journal of Econometrics 73, 151-184.

Brownlees, C.T. and G.M. Gallo (2006), Financial econometric analysis at ultra-high frequency: Data handling concerns, Computational Statistics and Data Analysis 51, 2232-2245.

Chiriac, R. and V. Voev (2011), Modelling and forecasting multivariate realized volatility, Journal of Applied Econometrics 26, 922-947.

Conrad, C. and B.R. Haag (2006), Inequality constraints in the fractionally integrated GARCH model, Journal of Financial Econometrics 4, 413-449.

Corsi, F. (2009), A simple approximate long-memory model of realized volatility, Journal of Financial Econometrics 7, 174-196.

Cox, D.R. (1981), Statistical analysis of time series: some recent developments, Scandinavian Journal of Statistics 8, 93-115.

Creal, D., S.J. Koopman and A. Lucas (2011), A dynamic Multivariate Heavy-Tailed Model for TimeVarying Volatilities and Correlations, Journal of Business and Economic Statistics 29, 552-563.

Creal, D., S.J. Koopman and A. Lucas (2013), Generalized Autoregressive Score Models with Applications, Journal of Applied Econometrics 28, 777-795.

Creal, D., S.J. Koopman, A. Lucas and B. Schwaab (2014), Observation Driven Mixed-Measurement Dynamic Factor Models with an Application to Credit Risk, Review of Economics and Statistics (forthcoming).

Diebold, F.X. and R.S. Mariano (1995), Comparing predictive accuracy, Journal of Business and Economic statistics 13, 253-263.

Engle, R. and B. Kelly (2012), Dynamic equicorrelation, Journal of Business \&6 Economic Statistics $\mathbf{3 0}(2), 212-228$.

Giacomini, R. and H. White (2006), Tests of conditional predictive ability, Econometrica 74(6), 1545-1578.

Golosnoy, V., B. Gribisch and R. Liesenfeld (2012), The conditional autoregressive Wishart model for multivariate stock market volatility, Journal of Econometrics 167, 211-223. 
Gourieroux, C., J. Jasiak and R. Sufana (2009), The Wishart Autoregressive process of multivariate stochastic volatility, Journal of Econometrics 150, 167-181.

Hansen, P.R., P. Janus and S.J. Koopman (2014), Modeling Daily Covariance: A Joint Framework for Low and High-frequency Based Measures, Working Paper.

Harvey, A.C. (2013), Dynamic Models for Volatility and Heavy Tails: With Applications to Financial and Economic Time Series, Cambridge University Press.

Harvey, A.C. and A. Luati (2014), Filtering with heavy tails, Journal of the American Statistical Association (forthcoming) .

Janus, P., S.J. Koopman and A. Lucas (2014), Long memory dynamics for multivariate dependence under heavy tails, Journal of Empirical Finance 29, 187-206.

Konno, Y. (1991), A Note on Estimating Eigenvalues of Scale Matrix of the Multivariate F-distribution, Annals of the Institute of Statistical Mathematics 43, 157-165.

Koopman, S.J., B. Jungbacker and E. Hol (2005), Forecasting daily variability of the S\&P 100 stock index using historical, realised and implied volatility measurements, Journal of Empirical Finance 12, 445-475.

Lucas, A., B. Schwaab and X. Zhang (2014), Conditional Euro Area Sovereign Default Risk, Journal of Business and Economic Statistics 32(2), 271-284.

Markowitz, H. (1952), Portfolio selection, Journal of Finance 7(1), 77-91.

Noureldin, D., N. Shephard and K. Sheppard (2012), Multivariate high-frequency-based volatility (HEAVY) models, Journal of Applied Econometrics 27, 907-933.

Oh, D.H. and A.J. Patton (2016), Time-Varying Systemic Risk: Evidence from a Dynamic Copula Model of CDS Spreads, Journal of Business and Economic Statistics (forthcoming) .

Opschoor, A., P. Janus, A. Lucas, and D. van Dijk (2017), New HEAVY models for fat-tailed realized covariances and returns, Journal of Business and Economic Statistics (forthcoming) .

Patton, A.J. (2011), Volatility forecast comparison using imperfect volatility proxies, Journal of Econometrics $\mathbf{1 6 0}(1), 246-256$.

Proietti, T (2016), Component-wise Representations of Long-memory Models and Volatility Prediction, Journal of Financial Econometrics .

Shephard, N. and K. Sheppard (2010), Realising the future: forecasting with high-frequency-based volatility (heavy) models, Journal of Applied Econometrics 25, 197-231. 
Silvennoien, A. and T. Teräsvirta (2009), Multivariate GARCH models, in T.G. Andersen, A. Davis, J.P. Kreib and T. Mikosch (eds.), Handbook of Financial Time Series, Springer-Verlag, pp. 201-229.

Zumbach, G. (2006), The RiskMetrics 2006 methodology, Working Paper. 


\section{Web Appendix to:}

Fractional Integration and Fat Tails for

Realized Covariance Kernels and Returns

Anne Opschoor ${ }^{a}$ and André Lucas ${ }^{a}$ 


\section{Appendix A: Cleaning high-frequency data}

We obtain intraday trade data (stock prices) of 15 stocks from the TAQ database during the period 20012014. The cleaning procedure stems from Barndorff-Nielsen et al. (2009) and Brownlees and Gallo (2006) and consists of the following steps:

1 Delete entries with a time stamp outside the 9:30 am4 pm window when the exchange is open.

2 Delete entries with a bid, ask or transaction price equal to zero.

3 Retain entries originating from a single exchange (NYSE in our application). Delete other entries.

4 Delete entries with corrected trades. (Trades with a Correction Indicator, CORR $\neq 0$ ).

5 Delete entries with abnormal Sale Condition. (Trades where COND has a letter code,except for "E" and "F"). See the TAQ 3 Users Guide for additional details about sale conditions.

6 If multiple transactions have the same time stamp, use the median price.

Note that the last three filters specifically correspond to Trade data only. After applying the above filters, we run the filter described in Brownlees and Gallo (2006) (see page 2237) that removes outliers. 


\section{Appendix B: Results of the EWMA model}

Table B.1: Test-statistics on predictive ability (FIGAS vs EWMA)

This table shows test statistics on superior predictive ability between the FIGAS tF model and long-memory EWMA model of Zumbach (2006), based on the QLIK loss function defined in (21). The test is based based on 1, 5, 10 and 22-step ahead predictions of the covariance matrix, applied to 5 and 15 (all) equities. Panel A present results for full out-of-sample (December 2006 - December 2012), Panel B describes the results for the Financial Crisis period (July 2007 - December 2009) $k=15$ and panel C shows the results for the Non-crisis period (Dec 2006 - July 2007 and January 2010 - December 2012). The subpanels 1 and 2 correspond to number of considered assets, i.e. $k=5$ and $k=15$. We report the average QLIK loss for both models with the associated DM-type of test statistic in parentheses. A negative test statistic indicates superior predictive ability of the FIGAS tF model. We use a moving window of 1500 observations. The prediction period contains 1495 observations.

\begin{tabular}{|c|c|c|c|c|c|c|c|}
\hline & 1 & 5 & 10 & 22 & $1: 5$ & $1: 10$ & $1: 22$ \\
\hline \multicolumn{8}{|c|}{ Panel A: Full out-of-sample } \\
\hline \multicolumn{8}{|c|}{ A.1: AA/BA/CAT/GE/KO } \\
\hline FIGAS & 8.04 & 8.42 & 8.68 & 9.12 & 16.30 & 19.93 & 24.16 \\
\hline \multirow{2}{*}{ EWMA } & 8.98 & 10.02 & 11.59 & 15.76 & 17.48 & 21.54 & 26.96 \\
\hline & $(-9.9)$ & $(-7.9)$ & $(-6.2)$ & $(-4.6)$ & $(-8.6)$ & $(-6.7)$ & $(-4.9)$ \\
\hline \multicolumn{8}{|c|}{ A.2: All assets $(k=15)$} \\
\hline FIGAS & 19.06 & 20.03 & 20.76 & 21.88 & 43.75 & 54.60 & 67.18 \\
\hline \multirow[t]{2}{*}{ EWMA } & 27.28 & 31.02 & 36.24 & 49.85 & 53.13 & 65.63 & 82.53 \\
\hline & $(-10.1)$ & $(-9.1)$ & $(-6.8)$ & $(-4.9)$ & $(-9.7)$ & $(-7.3)$ & $(-5.4)$ \\
\hline
\end{tabular}

Panel B: Crisis period

\begin{tabular}{llllllll}
\hline \multicolumn{8}{l}{ B.1: AA/BA/CAT/GE/KO } \\
\hline FIGAS & 10.82 & 11.38 & 11.86 & 12.71 & 19.17 & 22.92 & 27.34 \\
EWMA & 12.07 & 13.72 & 16.31 & 23.49 & 20.85 & 25.28 & 31.71 \\
& $(-7.4)$ & $(-5.6)$ & $(-4.4)$ & $(-3.4)$ & $(-6.1)$ & $(-4.8)$ & $(-3.6)$ \\
\hline B.2: All assets $(k=15)$ & & & & & \\
\hline FIGAS & 27.67 & 29.14 & 30.46 & 32.76 & 52.60 & 63.76 & 76.95 \\
EWMA & 40.34 & 46.66 & 55.50 & 79.50 & 67.38 & 81.41 & 102.0 \\
& $(-7.6)$ & $(-6.7)$ & $(-5.1)$ & $(-3.8)$ & $(-7.1)$ & $(-5.4)$ & $(-4.1)$ \\
\hline
\end{tabular}

Panel C: Non-Crisis period

\begin{tabular}{lccccccc}
\hline C.1: AA/BA/CAT/GE/KO & & & & \\
\hline FIGAS & 6.00 & 6.25 & 6.35 & 6.49 & 14.21 & 17.75 & 21.82 \\
EWMA & 6.72 & 7.31 & 8.13 & 10.10 & 15.02 & 18.79 & 23.48 \\
& $(-7.2)$ & $(-6.7)$ & $(-6.3)$ & $(-5.9)$ & $(-7.0)$ & $(-6.1)$ & $(-5.4)$ \\
\hline C.2: All assets $(k=15)$ & & & & & \\
\hline FIGAS & 12.76 & 13.35 & 13.67 & 13.92 & 37.28 & 47.89 & 60.03 \\
EWMA & 17.74 & 19.59 & 22.14 & 28.17 & 42.72 & 54.09 & 68.25 \\
& $(-9.9)$ & $(-9.4)$ & $(-8.0)$ & $(-7.2)$ & $(-9.9)$ & $(-8.3)$ & $(-7.0)$ \\
\hline
\end{tabular}


Table B.2: Ex-post minimum variance portfolio standard-deviations (FIGAS vs EWMA)

This table shows results on a global minimum variance portfolio, based on 1, 5, 10 and 22-step ahead predictions of the covariance matrix, according to the FIGAS tF and the (long-memory) EWMA model of Zumbach (2006). GAS, HEAVY and the multivariate HAR model, applied to 5 and 15 equities. For each model, the table shows the ex-post mean of the daily portfolio volatility, whereas below the GAS, HEAVY and HAR model. We use a moving window of 1500 observations. The prediction period runs from December, 2006 until December, 2012 (1495 observations).

\begin{tabular}{|c|c|c|c|c|c|c|c|}
\hline & 1 & 5 & 10 & 22 & $1: 5$ & $1: 10$ & $1: 22$ \\
\hline \multicolumn{8}{|c|}{ Panel A: AA/BA/CAT/GE/KO } \\
\hline FIGAS & 0.925 & 0.933 & 0.938 & 0.946 & 2.121 & 3.032 & 4.567 \\
\hline EWMA & $\begin{array}{c}0.983 \\
(-20.9)\end{array}$ & $\begin{array}{c}0.978 \\
(-11.7)\end{array}$ & $\begin{array}{l}0.975 \\
(-7.7)\end{array}$ & $\begin{array}{l}0.970 \\
(-5.3)\end{array}$ & $\begin{array}{c}2.235 \\
(-12.5)\end{array}$ & $\begin{array}{l}3.179 \\
(-9.1)\end{array}$ & $\begin{array}{l}4.749 \\
(-6.6)\end{array}$ \\
\hline \multicolumn{8}{|c|}{ Panel B: all equities } \\
\hline FIGAS & 0.688 & 0.700 & 0.707 & 0.718 & 1.589 & 2.282 & 3.461 \\
\hline EWMA & $\begin{array}{c}0.834 \\
(-25.0)\end{array}$ & $\begin{array}{c}0.819 \\
(-12.4)\end{array}$ & $\begin{array}{l}0.808 \\
(-9.0)\end{array}$ & $\begin{array}{l}0.795 \\
(-6.3)\end{array}$ & $\begin{array}{c}1.884 \\
(-12.5)\end{array}$ & $\begin{array}{l}2.663 \\
(-9.1)\end{array}$ & $\begin{array}{l}3.946 \\
(-6.3)\end{array}$ \\
\hline
\end{tabular}

Web Appendix p. 3 